\title{
Solid-State NMR Studies of Supercapacitors
}

\author{
John M. Griffin, ${ }^{1,2}$ Alexander C. Forse, ${ }^{1}$ Clare P. Grey ${ }^{1}$ \\ ${ }^{1}$ Department of Chemistry, University of Cambridge, Lensfield Road, Cambridge, \\ CB2 1EW, UK \\ ${ }^{2}$ Department of Chemistry, Lancaster University, Lancaster, LA1 4YB, UK
} email: j.griffin@lancaster.ac.uk, cpg27@cam.ac.uk

Submitted to Solid State Nuclear Magnetic Resonance (Trends Article) 


\begin{abstract}
Electrochemical double-layer capacitors, or 'supercapacitors' are attracting increasing attention as high-power energy storage devices for a wide range of technological applications. These devices store charge through electrostatic interactions between liquid electrolyte ions and the surfaces of porous carbon electrodes. However, many aspects of the fundamental mechanism of supercapacitance are still not well understood, and there is a lack of experimental techniques which are capable of studying working devices. Recently, solid-state NMR has emerged as a powerful tool for studying the local environments and behaviour of electrolyte ions in supercapacitor electrodes. In this Trends article, we review these recent developments and applications. We first discuss the basic principles underlying the mechanism of supercapacitance, as well as the key NMR observables that are relevant to the study of supercapacitor electrodes. We then review some practical aspects of the study of working devices using ex situ and in situ methodologies and explain the key advances that these techniques have allowed on the study of supercapacitor charging mechanisms. NMR experiments have revealed that the pores of the carbon electrodes contain a significant number of electrolyte ions in the absence of any charging potential. This has important implications for the molecular mechanisms of supercapacitance, as charge can be stored by different ion adsorption/desorption processes. Crucially, we show how in situ NMR experiments can be used to quantitatively study and characterise the charging mechanism, with the experiments providing the most detailed picture of charge storage to date, offering the opportunity to design enhanced devices. Finally, an outlook for future directions for solid-state NMR in supercapacitor research is offered.
\end{abstract}




\section{Introduction}

The development of efficient energy storage devices is essential to support our growing reliance on portable electronics, green transportation and renewable energy sources. While lithium-ion batteries have met a large proportion of our energy storage needs over the past 20 years, electrochemical capacitors, also known as supercapacitors, are currently receiving renewed interest as an alternative or complementary technology. ${ }^{1}$ Supercapacitors store energy through the formation of an electrical double-layer at the interface between ions in a liquid electrolyte and a microporous carbon electrode surface. This non-faradaic charge storage mechanism allows rapid charge and discharge rates with essentially unlimited cycle lives, making supercapacitors wellsuited to high-power and/or fast-repeat applications such as electric buses, subway trains and cranes. $^{2}$

While supercapacitors display many favourable properties, current gravimetric energy densities remain at least an order of magnitude lower than lithium ion batteries. ${ }^{3}$ This makes them currently unsuitable as a sole power source for many applications e.g., electric cars. One of the ongoing challenges in the development of supercapacitors is therefore to increase the device energy density while retaining high power performance. Of critical importance is the characterization of the local environments and behaviour of adsorbed ions at the electrode-electrolyte interface, and to understand how this changes during charging. However, this information is very challenging to obtain experimentally. Supercapacitor electrodes are highly disordered and dynamic on the molecular level, and adsorbed species can be difficult to distinguish from free electrolyte species. Many forms of spectroscopy are unable to penetrate far into the porous network and conventional scattering techniques provide only limited information owing to the disorder and dynamics that are present. In this regard, solid-state NMR spectroscopy stands out as one of the few experimental techniques that can provide atomic-scale information about the local environments of adsorbed species. The fact that NMR is sensitive to local effects and has no requirement for crystallinity or long-range ordering means that adsorbed species can be observed within the highly complex porous structure, and those close to the electrode surface can be distinguished from free species in the bulk electrolyte. The highly selective nature of NMR means that experiments can be designed to observe only a single molecular, ionic or solvent species independently from the rest of the system. Furthermore, the ability of NMR to provide information on dynamics over a large range of timescales means that the behavior of adsorbed species can be monitored and linked to the structure and properties of the electrode. 

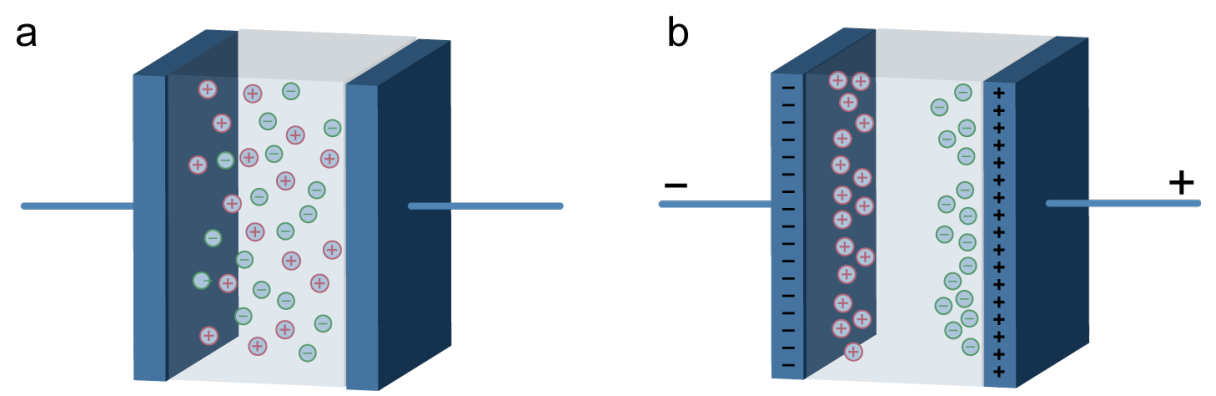

Figure 1. Schematic representation of the mechanism of supercapacitance. In the uncharged state (a), the supercapacitor consists of two conducting electrodes separated by a liquid electrolyte containing mobile ions. During charging (b), electronic charge accumulated on the electrode surface and is balanced by ionic charge that adsorbs on the electrode surface.

The unique capabilities of NMR spectroscopy for studying supercapacitors have contributed to a number of recent breakthroughs that challenge longstanding assumptions about the supercapacitance phenomenon, and show that the behaviour of electrolyte ions within microporous electrodes is more complex than previously thought. In particular, NMR experiments have proved that significant numbers of electrolyte ions exist within the micropores prior to charging. This has important implications for the mechanism of charge storage, which has traditionally been thought of as a purely adsorptive process. Indeed, recent NMR studies have also shown that ion desorption also plays an important role and the exact mechanism depends on the properties of the electrolyte ions used. In this Trends article, we review recent developments and applications of solid-state NMR spectroscopy for the study of supercapacitors and show how these important conclusions have been drawn from experimental observations. We begin with an overview of the basic principles of supercapacitors and the materials used in devices. This is followed by an explanation of key NMR observables that enable the identification of adsorbed species and an overview of how solid-state NMR has been used to study ion adsorption and local structure in supercapacitor electrodes in the recent literature. Finally, we present a discussion of the recent development of solid-state NMR methods for the study of charging mechanisms in working supercapacitor devices, and an outlook for future directions

\section{Background to Supercapacitors}

\subsection{Basic principles}

There are two main families of supercapacitors. The first is electrochemical double-layer capacitors which, as described in the introduction, store charge through the formation of an electrical double layer at the interface between a liquid electrolyte and a porous carbon electrode surface. Another emerging family is so-called pseudocapacitors, where the mechanism of energy storage is based upon redox reactions between electrolyte ions and (typically) the surface of an oxide electrode. ${ }^{4}$ Pseudocapacitors show considerable promise as energy storage devices, since the faradaic component of the charge storage mechanism can significantly increase the total amount of energy 
stored. Fundamental mechanistic studies of pseudocapacitors remain scarce and the details of the complex charge transfer mechanisms are only just beginning to be understood. ${ }^{5}$ We do not cover pseudocapacitors in detail in this Trends article, and instead use the term 'supercapacitor' to refer specifically to electrochemical double-layer capacitors.

Supercapacitors typically consist of two porous carbon electrodes separated by liquid electrolyte containing mobile ions. ${ }^{6,7}$ The basic operation of a supercapacitor is shown schematically in Figures $1 \mathrm{a}$ and $\mathrm{b}$. Upon charging, electronic and ionic charges accumulate at the electrodeelectrolyte interface to form an electrical double layer. The capacitance, $C$, of such a "parallel plate" electrical double layer can be described by:

$$
C=\frac{\varepsilon_{r} \varepsilon_{0} A}{d}
$$

where $\varepsilon_{\mathrm{r}}$ is the relative permittivity of the electrolyte, $\varepsilon_{0}$ is the permittivity of free space, $A$ is the interfacial area and $d$ is the distance between charges in the electrical double layer. ${ }^{6}$ The energy stored, $E$, is given by

$$
E=\frac{1}{2} C V^{2}
$$

where $V$ is the cell voltage. The energy stored is therefore dependent on two basic parameters: the capacitance (which in turn depends upon the structure and properties of the electrode-electrolyte interface) and the voltage across the electrode-electrolyte interface. Optimisation of both of these parameters is key to maximizing the energy storage properties of supercapacitors.

\subsection{Supercapacitor electrolytes}

Supercapacitors typically use organic electrolytes, such as tetraethylammonium tetrafluoroborate $\left(\mathrm{NEt}_{4}-\mathrm{BF}_{4}\right)$ salt dissolved in a solvent such as acetonitrile $(\mathrm{ACN})$ or propylene carbonate. ${ }^{1,},{ }^{8}$ These electrolytes are favoured owing to their moderate breakdown voltage $(\sim 2.7 \mathrm{~V})$ and high ionic conductivities. Aqueous electrolytes can also be used - these have the advantages of being cheaper and less toxic; ${ }^{9}$ however, the decomposition of water at voltages above $1.2 \mathrm{~V}$ significantly reduces the amount of energy that can be stored. Recently, room-temperature ionic liquids (RTILs) have gained attention as alternatives to organic electrolytes. ${ }^{10},{ }^{11},{ }^{12},{ }^{13}$ RTILs have a number of attractive properties including low vapour pressure, nonflammability, and good chemical and thermal stabilities. Most importantly, they have significantly wider electrochemical stability windows of up to $5 \mathrm{~V} .{ }^{12}$ However, their widespread use as supercapacitor electrolytes has so far been 
limited due to their high viscosities which limit the power capabilities of devices, although current research is attempting to address this problem. ${ }^{14},{ }^{15},{ }^{16}$

\subsection{Supercapacitor electrode materials}

Supercapacitor electrode materials need to be highly conductive to allow transport of electronic charge to the electrode-electrolyte interface, and high surface areas are required to maximize the capacitance according to Eq. 1. For these reasons, electrodes are typically constructed from porous carbon. ${ }^{1,},{ }^{17}$ While many forms of porous carbon exist and continue to be developed, microporous carbons (with pore widths less than $2 \mathrm{~nm}$ ) are favoured owing to the high surface areas that these materials can exhibit. In particular, activated carbons derived from organic precursors such as coconut shells and wood are widely used in commercial devices owing to their high conductivity, high surface area (up to $2000 \mathrm{~m}^{2} \cdot \mathrm{g}^{-1}$ ) and low cost. These carbons are predominantly microporous, although they also exhibit a range of pore diameters in the mesoporous $(2-50 \mathrm{~nm})$ and macroporous $(>50 \mathrm{~nm}$ ) regimes which can significantly aid the movement of electrolyte species through the porous network.

Microporous carbon materials are generally non-crystalline, with highly disordered or amorphous structures that are difficult to characterize. While some templated carbon materials can exhibit highly ordered pore structures, these also generally lack long-range ordering of carbon atoms. One of the key techniques that has been used to gain structural insight into microporous carbons is transmission electron microscopy (TEM). TEM images generally show relatively open structures comprising voids that are surrounded by curved and twisted carbon sheets. ${ }^{18}$ The structures are isotropic with no overall alignment of the carbon sheets (in contrast to graphitic materials where the sheets pack along a preferred orientation). While microporous carbons appear fully amorphous by conventional scattering techniques, pair-distribution function (PDF) analysis has shown that they can exhibit a high degree of local structural order, whereby carbon atoms are predominantly hexagonally arranged in $s p^{2}$-hybridised six-membered rings. ${ }^{19},{ }^{20}$, The curvature of the carbon sheets is thought to be related to the presence of small numbers of non-hexagonal five- and seven-membered rings which have been observed in experimental studies and predicted by modeling techniques. ${ }^{20},{ }^{21},{ }^{22}$ There has been considerable debate over the presence of $s p^{3}$ hybridised carbon atoms in microporous carbon materials. However, experimental studies based on neutron diffraction, ${ }^{23}$ Raman spectroscopy ${ }^{24}$ and ${ }^{13} \mathrm{C}$ solid-state $\mathrm{NMR}^{25},{ }^{26}$ indicate that most microporous carbon materials consist of carbon predominantly in $s p^{2}$-hybridised environments.

Overall, the structural characterization of microporous carbons is challenging and remains an active area of research. Experimental and theoretical research to date points towards a general 
structural picture that comprises curved and defective graphene- and fullerene-like fragments. ${ }^{18}$ The high porosity is formed by the voids and spaces between the carbon sheets. Several experimental and theoretical studies indicate that the average size of the sheets is linked to the synthesis conditions, with higher temperatures leading to larger, more ordered fragments in a more continuous network. ${ }^{27},{ }^{28},{ }^{29}$

\subsection{Pore size effects on capacitance}

The use of microporous carbon electrodes has important implications for the fundamental mechanism of supercapacitance. In many materials, the majority of pore widths are less than 2 $\mathrm{nm}$, making them comparable to the diameters of solvated electrolyte ions. In this case, adsorbed ions can no longer be treated as being adsorbed against a single interface (as in Figure 1b), but are instead better described as being confined between two surfaces. Interest in understanding the relationship between capacitance and relative pore/ion sizes was prompted by a key study by Chmiola et al., who reported that the capacitance of titanium carbide-derived carbons (TiC-CDCs) dramatically increased when the average size of carbon micropores was less than the size of the solvated electrolyte ions (typically less than $1.5 \mathrm{~nm}$ ). ${ }^{30}$ It was hypothesized that ion desolvation allowed a closer approach of charge centers at the electrode-electrolyte interface, thereby reducing the separation of charges within the electrical double layer (parameterized by $d$ in Eq. 1). ${ }^{31},{ }^{32}$ Since then, a large number of theoretical studies have shown that the charge storage mechanism in microporous electrodes may be more complex, with factors such as charge screening, ${ }^{33},{ }^{34},{ }^{35}$ ionic rearrangement and confinement, ${ }^{36},{ }^{37}$ and pore surface properties ${ }^{38}$ also having significant effects on the capacitance and charging dynamics.

The effects of relative pore/ion size on the capacitance, and the solvation states of ions in charged micropores are still under debate. Much of the current picture of supercapacitor charging has come from theoretical studies which, owing to the high complexity of the system, necessarily involve assumptions and simplifications. Experimental insight is difficult to obtain due to the inherent difficulty in distinguishing electrolyte species taking part in charge storage from those in the bulk electrolyte, and a lack of techniques which can provide molecular-level information for working devices. However, as is described in the following sections, solid-state NMR spectroscopy is wellsuited to study these problems, and has recently emerged as an important tool for gaining structural and mechanistic insight into supercapacitor charging mechanisms. 


\section{Background to solid-state NMR spectroscopy for the study of supercapacitors}

The great utility of NMR spectroscopy for the study of supercapacitors lies in the high sensitivity of nuclear spin precession frequencies to the local chemical and structural environment. By performing NMR experiments on the nuclear spins present in electrolyte species, they can be made to serve as valuable probes or "reporter molecules", providing valuable information about the electrode structure, electrolyte behavior and charging mechanisms. In this section we briefly introduce some of the key experimental techniques used to carry out NMR experiments on supercapacitor materials, as well as the nuclear spin properties of commonly-studied nuclei. We then discuss in more how NMR can be used to distinguish adsorbed species from those in the bulk electrolyte.

\subsection{Commonly-used techniques for the study of supercapacitors by solid-state NMR}

Magic-angle spinning. Nuclear spins are subject to a variety of magnetic interactions with the local electron distribution and other nearby nuclear spins. In general, nuclear spin interactions are anisotropic with respect to the applied magnetic field direction, and for complex and disordered materials such as supercapacitor electrodes, this leads to broadening of the resonances in the NMR spectrum, and a loss of resolution. To mitigate this problem, magic-angle spinning (MAS) can be employed, whereby the sample (e.g., activated carbon soaked with electrolyte, electrodes from disassembled supercapacitor cells etc.) is rapidly spun around an axis at the "magic" angle of $54.736^{\circ}$ to the applied magnetic field. Providing the rotation is sufficiently rapid, the resulting modulation of the NMR signal enables removal of anisotropic interactions such as the chemical shift anisotropy, dipolar couplings and isotropic magnetic susceptibility effects (i.e., those which have an orientation dependence proportional to the second-order Legendre polynomial $P_{2}(\cos \theta)=$ $1 / 2\left(3 \cos ^{2} \theta-1\right)$. For spin $I=1 / 2$ nuclei, in many cases MAS results in the observation of "isotropic" spectra, containing sharp resonances at the isotropic chemical shift, which are surrounded by spinning sidebands which are equally spaced at integer multiples of the rotation frequency from the isotropic resonance. In many cases, it is desirable to perform NMR experiments at a MAS rate that is larger than the magnitude of the anisotropic interaction so that the signal intensity is "focused" into a single isotropic resonance, thereby maximising sensitivity. However, in practice MAS experiments on supercapacitor electrodes are performed at relatively low MAS rates (a few $\mathrm{kHz}$ ) in order to minimize leakage of the liquid electrolyte from the sample due to centrifugal forces, ${ }^{39}$ and consequent damage to the NMR equipment. We note that for NMR studies of supercapacitor electrolyte species, the mobility of the electrolyte species is often sufficient to partially average the anisotropic interactions that are present, and good resolution can be obtained in the absence of MAS (i.e., in "static" NMR measurements). However, for electrolytes with reduced mobility (e.g., ionic liquids which have higher viscosities), the molecular motion at ambient temperatures is not 
sufficient to yield well-resolved spectra and MAS is usually necessary in order to obtain detailed information.

Cross polarisation. The simultaneous application of radiofrequency irradiation to two different types of nuclear spin can result in polarisation transfer via the dipole-dipole coupling interaction. This approach, referred to as cross polarisation (CP), is widely used in solid-state NMR to achieve polarisation enhancement of low sensitivity nuclei, as the transfer process also results in a polarisation enhancement equal to the ratio of the gyromagnetic ratios of the two nuclei. However, since the transfer process is mediated via the through-space dipolar coupling interaction, CP also provides a powerful technique for correlating spins that are close in space. Since the dipolar coupling interaction is inversely proportional to the cube of the nuclear spin separation distance, CP generally acts over relatively short distances, on the order of a few angstroms in most materials. CP can therefore be used to identify which pairs of heteronuclei are close together in space (or bonded), and also to edit NMR spectra by removing signals from spins that have no nearby heteronuclei. Generally CP is most effective for studying solid materials, as in liquids molecular tumbling and rotation average the dipolar couplings to zero. In solids with considerable molecular motion (or indeed liquids which are confined or immobilised) CP can still be achieved in many cases, and the dynamics of the polarisation transfer can be used as a probe of molecular motion. $^{40}$

Magnetic resonance imaging. Magnetic resonance imaging (MRI) encodes spatial resolution into the NMR measurement through the application of magnetic field gradients across the sample. While this technique is widely established in medical diagnostics, it is seeing increasing application to energy materials including batteries, fuel cells and supercapacitors. In conventional MRI, twodimensional slices or three-dimensional visualisations of the sample are built up, often through the measurement of differences in nuclear spin relaxation properties between the different components of the sample. More specific chemical information can be obtained through the application of chemical shift imaging (CSI). This approach spatially encodes chemical shift information in one or two dimensions so that it can be correlated with different regions of the sample. Chemical shift images therefore offer the possibility to obtain detailed chemical information about distinct components within a supercapacitor without the need for disassembly or special design of the device. However, owing to the typically longer durations of MRI experiments as compared to conventional NMR experiments, nuclear spin relaxation can be significant and it is therefore not always possible to obtain fully quantitative information. 


\subsection{Commonly studied nuclei in supercapacitors}

${ }^{1} \mathrm{H}$. ${ }^{1} \mathrm{H}$ is in principle a very favourable nucleus for study by NMR as it has an almost $100 \%$ natural abundance, high sensitivity, and is present in many supercapacitor electrolyte and solvent species. However, ${ }^{1} \mathrm{H}$ also has a relatively small chemical shift range, with most commonly encountered ${ }^{1} \mathrm{H}$ environments giving resonances within a range of $\sim 15 \mathrm{ppm}$ or less. In practice, this can lead to resonance overlap if more than one chemically-distinct species is present in the sample (e.g., $\mathrm{CH}_{2}$ and $\mathrm{CH}_{3}$ groups in alkylammonium electrolyte salts). Another problem associated with the study of supercapacitors by ${ }^{1} \mathrm{H}$ NMR is that ${ }^{1} \mathrm{H}$ is present in many supercapacitor components (e.g., the separator or cell casing materials used for in situ measurements), and strong background signals from these can hinder the observation and interpretation of resonances corresponding to the electrolyte species. That said, the high sensitivity of ${ }^{1} \mathrm{H}$ still makes it a highly valuable probe, especially if the aforementioned challenges can be overcome.

${ }^{19} \mathrm{~F} .{ }^{19} \mathrm{~F}$ is a highly sensitive nucleus with a $100 \%$ natural abundance making it highly favourable for NMR studies of supercapacitors. ${ }^{19} \mathrm{~F}$ has a large chemical shift range ( 200 ppm) meaning that different chemical species are well resolved and easily distinguished. Furthermore, in many electrolyte systems ${ }^{19} \mathrm{~F}$ is present in a high molar ratio (e.g., in $\mathrm{BF}_{4}$ or $\mathrm{CF}_{3}$ groups), which also increases the sensitivity. The use of PTFE of PVDF polymer binders in supercapacitor electrodes can lead to broad background signals in the NMR spectrum; however, these usually have much shorter relaxation times than the electrolyte species, and the background signals can be effectively removed using spin-echo or common background suppression NMR pulse sequences.

${ }^{11} \mathrm{~B}$. The ${ }^{11} \mathrm{~B}$ nucleus has a nuclear spin $I=3 / 2$ and is therefore subject to quadrupolar interactions which can potentially lead to broadening of spectral resonances for non-symmetric local environments. However, in supercapacitor electrolytes the ${ }^{11} \mathrm{~B}$ is usually encountered in $\mathrm{BF}_{4}{ }^{-}$ anions which have a high degree of structural symmetry. In addition to this, the fast isotropic motion of the $\mathrm{BF}_{4}^{-}$anions at ambient temperatures is also sufficient to average any residual local structural asymmetry. Compared to ${ }^{1} \mathrm{H}$ and ${ }^{19} \mathrm{~F},{ }^{11} \mathrm{~B}$ has a lower sensitivity owing to a lower natural abundance (80\%) and lower gyromagnetic ratio, and longitudinal relaxation times can be relatively long in some systems (tens of seconds or minutes) meaning that quantitative NMR data takes longer to acquire.

${ }^{31} \mathrm{P} .{ }^{31} \mathrm{P}$ is also a favourable nucleus for the study of supercapacitor electrodes as, like ${ }^{19} \mathrm{~F}$, it has a $100 \%$ natural abundance and a large chemical shift range, and has a nuclear spin $I=1 / 2$. In addition, it is not present in typical supercapacitor components, meaning that background signals are generally not observed. As described in Section $5.5,{ }^{31} \mathrm{P}$ NMR provides a valuable probe of 
cation environments in phosphonium-based electrolytes, enabling the fully quantitative study of supercapacitor electrolyte ions.

${ }^{13} \mathrm{C} .{ }^{13} \mathrm{C}$ is present in most organic electrolytes, solvents, and ionic liquids, and ${ }^{13} \mathrm{C} N M R$ can in principle provide valuable chemical information owing to the large chemical shift range (200 ppm). However, the main practical challenge associated with ${ }^{13} \mathrm{C} N \mathrm{NR}$ is the low sensitivity, which is primarily due to the low natural abundance of ${ }^{13} \mathrm{C}(1.1 \%)$. Despite this, ${ }^{13} \mathrm{C}$ ex situ MAS NMR has been used for the study of organic electrolyte and solvent environments in supercapacitor electrodes $^{41}$ (as described in section 5.3). Furthermore, the use of materials that are isotopically enriched in ${ }^{13} \mathrm{C}$ can facilitate spectral acquisition by increasing the sensitivity, and also create new possibilities for selective experiments that are not possible at natural abundance (as described in section 6).

${ }^{2} \mathrm{H}$. The low natural abundance of ${ }^{2} \mathrm{H}(0.0156 \%)$ means that means that NMR studies are generally restricted to materials that have been isotopically enriched. However, as for ${ }^{13} \mathrm{C}$, this can enable the selective observation of specific chemical groups or molecular species. In particular, deuterated solvents are relatively inexpensive, and if used in supercapacitor electrolytes can be observed independently from the rest of the species in the system by ${ }^{2} \mathrm{H}$ NMR. Another advantage of ${ }^{2} \mathrm{H}$ NMR is that it can in principle provide detailed information about solid-state molecular motion over wide range of timescales; ${ }^{42}$ this could be useful for studying molecular dynamics for confined species in supercapacitor electrodes.

${ }^{23} \mathrm{Na} .{ }^{23} \mathrm{Na}$ is a favourable nucleus for study by NMR owing to its $100 \%$ natural abundance and high sensitivity. Like ${ }^{11} \mathrm{~B}$, it has a nuclear spin $I=3 / 2$ meaning that it is subject to quadrupolar interactions in non-symmetric environments. However, the quadrupole moment (which scales the quadrupolar interaction) is relatively small and when present in (e.g., aqueous) supercapacitor electrolytes, ionic motion is usually sufficient to average any local structural asymmetry so that resonances are resolved and distinct environments can be observed.

\subsection{Identifying adsorbed species in microporous carbon}

One of the key capabilities of NMR spectroscopy for the study of supercapacitors is the ability to distinguish electrolyte species adsorbed to the carbon surfaces inside the micropores (referred to as 'in-pore') from those in larger voids and spaces outside of the carbon particles (referred to as ex-pore). In-pore and ex-pore species can be identified in a simple adsorption experiment, as illustrated in Figure 2a. In this example, ${ }^{19} \mathrm{~F}$ static NMR spectra are shown for model activated carbon electrodes loaded with different amounts of $1.5 \mathrm{M} \mathrm{NEt}_{4}-\mathrm{BF}_{4} / \mathrm{ACN}$ electrolyte. ${ }^{43}$ For the 
a

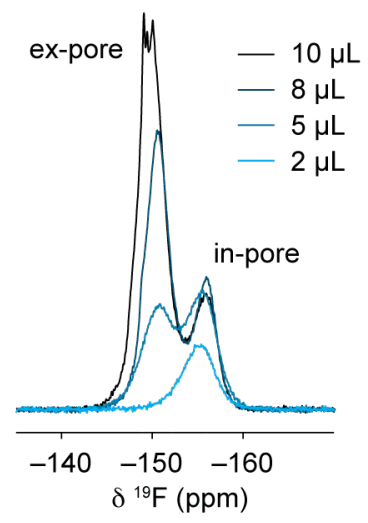

C

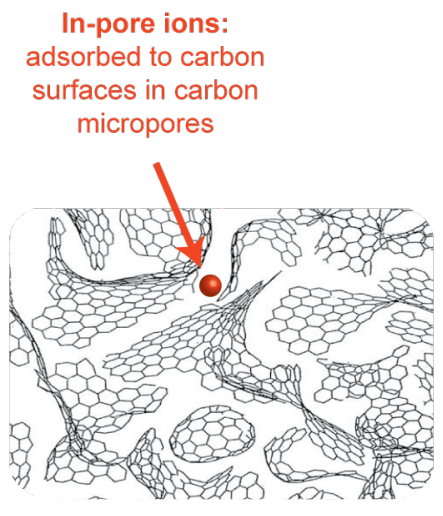

b

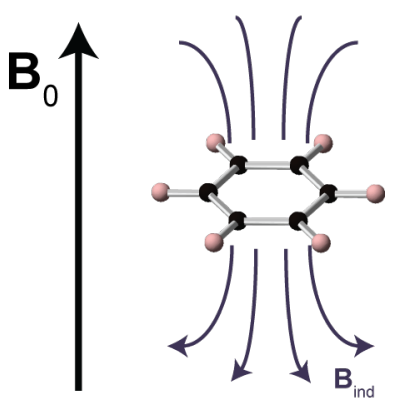

d

Ex-pore ions: in reservoirs of electrolyte in large spaces in carbon

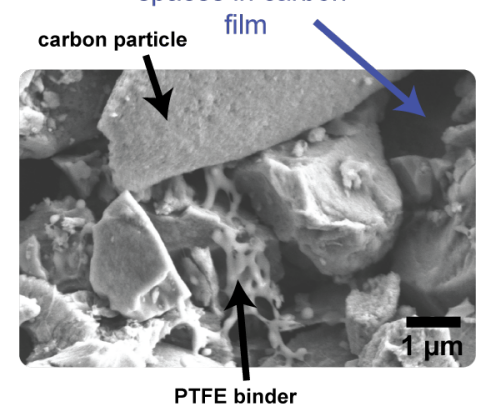

Figure 2. (a) ${ }^{19} \mathrm{~F}$ static NMR spectra of YP-50F activated carbon electrode films loaded with different amounts of NEt ${ }_{4}$ $\mathrm{BF}_{4} / \mathrm{ACN}$ electrolyte [Reproduced from Ref. ${ }^{43}$ with permission from The Royal Society of Chemistry.]. (b) Schematic illustration of the ring current-induced magnetic field, $\mathbf{B}_{\text {ind }}$, associated with delocalized $\pi$-electrons in a six-membered carbon ring within an applied magnetic field $\mathbf{B}_{0}$. (c) A cartoon showing in-pore ions located close to carbon surfaces inside the porous structure [Image reproduced from Ref. 16 with permission from Taylor \& Francis Ltd, http://www.tandfonline.com]. (d) SEM image showing voids between carbon particles in an electrode film where ex-pore electrolyte ions reside.

lowest loading volume of $2 \mu \mathrm{L}$, a single resonance is observed at $-155 \mathrm{ppm}$. As more electrolyte is added, the intensity of this resonance increases but then saturates around a loading volume of 5 $\mu \mathrm{L}$. Simultaneously, a second resonance is observed at $-150 \mathrm{ppm}$ which continues to grow in intensity as the electrolyte volume is increased.

The two resonances can be interpreted on the basis of their changes in intensity as a function of loading volume. The appearance, growth and saturation in intensity of the resonance at $-155 \mathrm{ppm}$ suggests the preferential occupation of in-pore adsorption sites within micropores by $\mathrm{BF}_{4}$ anions, where the adsorption sites become fully saturated at a loading volume of $\sim 5 \mu \mathrm{L}$. The subsequent appearance and growth of the resonance at $-150 \mathrm{ppm}$ is consistent with ex-pore $\mathrm{BF}_{4}$ anions occupying sites outside of the micropores in intergranular voids between the carbon particles. Indeed, this ex-pore resonance has an almost identical chemical shift to that of the pure liquid 
electrolyte. As more electrolyte is added, the ex-pore resonance at $-150 \mathrm{ppm}$ continues to grow because the extra-particulate space is effectively unlimited.

These observations are consistent with a number of early NMR studies of microporous carbon adsorbents which found that resonances corresponding to adsorbed species were shifted to low frequency by several ppm as compared to free species. ${ }^{44}, 45,46,47,48$ Similar observations have also been reported for species adsorbed within carbon nanotubes. ${ }^{49},{ }^{50}$ The origin of the pronounced NMR frequency shift for in-pore species lies in the so-called "ring current" effect that arises due to the presence of delocalized electrons in the $s p^{2}$-hybridised carbon surface. ${ }^{51}$ Application of the external magnetic field, $\mathbf{B}_{0}$, induces electron circulation within the delocalized $\pi$-orbitals, resulting in a secondary local magnetic field above the carbon surface that opposes the main field (as represented in Figure 2b). Nuclear species located nearby the delocalized electron system will therefore experience a locally altered magnetic field, and will therefore exhibit a resonance frequency shift in the NMR spectrum. Ring current-induced local magnetic fields can be diamagnetic or paramagnetic depending on the electronic structure of the delocalized electron system. Since microporous carbons are predominantly composed of six-membered rings with paired electrons, the ring current-induced local magnetic field is in general diamagnetic, meaning that species near the carbon surface are shielded and shifted to lower frequency in the NMR spectrum.

The magnitude of the ring current shift near an $s p^{2}$-hybridised carbon surface can be estimated using a phenomenological model taking into account the magnetic susceptibility. ${ }^{52}$ This will also have a dependence on the orientation of the carbon surface with respect to the applied magnetic field $\mathbf{B}_{0}$; however, for a gas or liquid adsorbate, molecular kinetics are in general fast enough that adsorbed species should experience many surface orientations on a timescale that is fast compared to the associated variations in NMR parameters. On this basis, Anderson et al., derived a relationship for the averaged chemical shift change, $\delta$, given by ${ }^{52}$

$$
\delta=\frac{2}{34 \pi}\left(\chi_{\|}-\chi_{\perp}\right) \frac{1}{z^{3}}
$$

where $\chi_{\|}$and $\chi_{\perp}$ are the respective molar magnetic susceptibilities of the surface parallel and perpendicular to the applied field, and $z$ is the distance above the carbon surface. Taking known values for graphite of $\chi_{\|}=-3.2 \times 10^{-9}$ and $\chi_{\perp}=-7.5 \times 10^{-11} \mathrm{~m}^{3} \cdot \mathrm{mol}^{-1}$ at room temperature, ${ }^{53}$ a value of $\delta=-10.2 \mathrm{ppm}$ is obtained at a distance of $3 \AA$ from the carbon surface. This value is in good agreement with shieldings of between -5 and -10 ppm typically observed for in-pore species in microporous carbons. 
Ring current effects therefore provide a very useful tool for the study of adsorbed species in supercapacitor electrodes. The shift in NMR frequency of nuclei in in-pore species close to the carbon surface (Figure 2c) is sufficient that in many cases adsorbed species are resolved from free species in the bulk electrolyte that reside outside of the micropores or between carbon particles (Figure 2d). Importantly, the intensities of in-pore resonances can be used to provide a quantitative measure of the number of species adsorbed inside the micropores. Furthermore, assuming there is no direct chemical interaction between the adsorbate species and the carbon surface, the ring current effect is nucleus independent to first approximation; i.e., the chemical shift of any nucleus will be shifted by a fixed amount which depends only on its location with respect to the carbon surface. In this way, it is also possible to separately study the different species present near the carbon surface (i.e., cations, anions and solvent molecules), something which is very challenging by other experimental methods. 


\section{Ion behaviour in supercapacitor electrodes}

\subsection{Understanding pore size effects on electrolyte adsorption}

In order to characterize charging mechanisms in supercapacitors it is essential to have a detailed picture of how electrolyte ions are adsorbed in an uncharged electrode as this provides the starting point for understanding the changes that take place during charging. In particular, it is important to understand how relative pore/ion sizes affect how ions are adsorbed, and whether ions are adsorbed in their solvated or desolvated states. TiC-CDCs offer a convenient model system for studying pore-size effects on adsorption behaviour. TiC-CDCs are synthetic microporous carbons produced by chlorine treatment of titanium carbide and are known to exhibit relatively uniform pore size distributions. ${ }^{54}, 55,24$ Moreover, control of the chlorination temperature during synthesis allows fine-tuning of the average pore size in the range 0.6 to $1.1 \mathrm{~nm}$, with higher temperatures resulting in a larger average pore size. ${ }^{54}$ Comparison of adsorption behaviour for TiC-CDCs produced at different temperatures therefore allows pore-size effects to be investigated.

${ }^{11} \mathrm{~B}$ NMR has been used to study electrolyte adsorption in TiC-CDCs chlorinated at 600,800 and $1000{ }^{\circ} \mathrm{C}$, with average pore sizes $0.81,0.92$ and $0.93 \mathrm{~nm}$, respectively. ${ }^{56}$ Static NMR experiments were performed on plastic bag samples containing pieces of TiC-CDC electrode film loaded with different volumes of $\mathrm{NEt}_{4}-\mathrm{BF}_{4} / \mathrm{ACN}$ electrolyte. The results (Figure 3) show characteristic adsorption behaviour for each sample, whereby $\mathrm{BF}_{4}$ anions preferentially occupy sites close to the carbon surface (as indicated by ${ }^{11} \mathrm{~B}$ ring current shifts of around $-5 \mathrm{ppm}$ ). However, saturation of the in-pore environment is reached at lower loading volumes for samples with smaller pore sizes, with complete saturation of the in-pore environment for TiC-CDC-600 at the lowest loading volume of $2 \mu \mathrm{L}$. This was rationalized by comparing the average and maximum pore diameters for each TiC-CDC sample with the solvated and desolvated anion diameters. TiC-CDC-600 is the only material for which both the maximum and average pore sizes are smaller than the solvated $\mathrm{BF}_{4}$ anion diameter of $1.16 \mathrm{~nm}$. The fact that TiC-CDC-600 does not show a high adsorption capacity suggests that spontaneous electrolyte adsorption in uncharged TiC-CDC electrodes involves ions in at least a partially solvated state. Since the majority of micropores in TiC-CDC-600 are smaller than the solvated ion size, adsorption is restricted and the surface becomes saturated at a low electrolyte loading volume. 

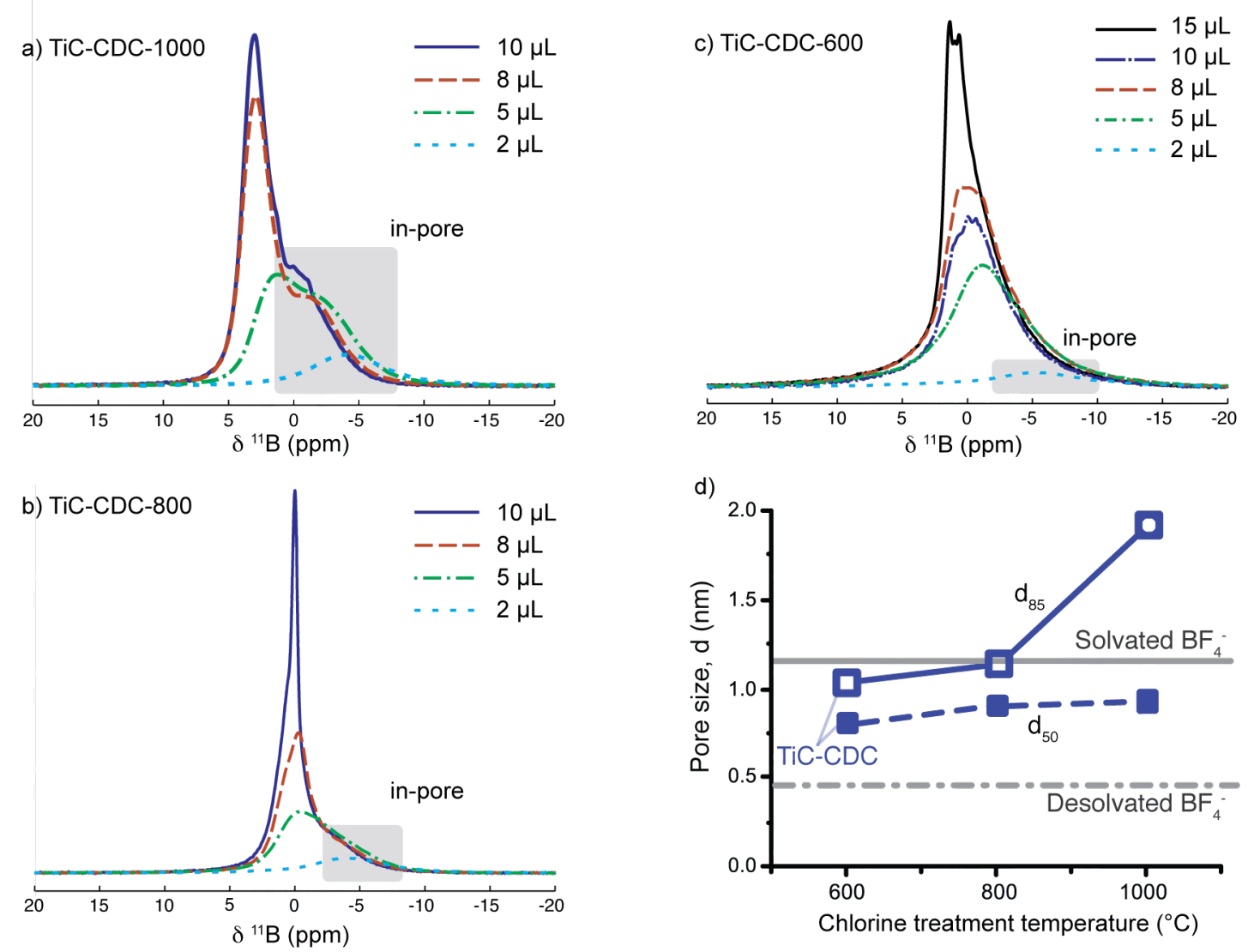

Figure 3. ${ }^{11} \mathrm{~B}$ static NMR spectra of (a) TiC-CDC-1000, (b) TiC-CDC-800 and (c) TiC-CDC-600 electrode films loaded with different amounts of $\mathrm{NEt}_{4}-\mathrm{BF}_{4} / \mathrm{ACN}$ electrolyte. (d) Comparison of average pore sizes, $\mathrm{d}_{50}$, and maximum pore sizes, $\mathrm{d}_{85}$, of TiC-CDCs with desolvated and solvated $\mathrm{BF}_{4}$ anion sizes. ${ }^{31}$ [Adapted from Ref. ${ }^{56}$ with permission from the PCCP Owner Societies].

In addition to the adsorption behaviour, the magnitude of the ring current shift can also be used to gain insight into the local environments and behaviour of in-pore electrolyte species. This has been demonstrated using ${ }^{11} \mathrm{~B},{ }^{1} \mathrm{H}$ and ${ }^{13} \mathrm{C}$ MAS NMR for the case of $\mathrm{NEt}_{4}-\mathrm{BF}_{4} / \mathrm{ACN}$ electrolyte adsorbed on porous carbons with a wider range of pore sizes from 0.6 to $4.5 \mathrm{~nm}$ (Figure $4 \mathrm{a}$ ). ${ }^{57}$ The results reveal an inverse relationship between the magnitude of the ring current shift and the pore diameter for all electrolyte species, consistent with a previous ${ }^{1} \mathrm{H}$ NMR study of $\mathrm{H}_{2}$ gas adsorption in activated carbons. ${ }^{52}$ This effect was interpreted in terms of a spherical pore model (Figure $4 \mathrm{~b}$ ), where the measured ring current shift corresponds to the weighted averaged of species near the pore wall and in the centre of the pore. ${ }^{57}$ In larger pores, more of the ions are located further away from the pore walls, and so the averaged ring current shift is smaller. This model gives good agreement with the experimental observations and shows that in-pore species are highly mobile within the pores, such that the variation in ring current shift at different distances from the pore wall is averaged on the timescale of the NMR experiment. This is also true for a CDC with a bimodal pore size distribution, for which only a single in-pore resonance is observed, showing that the fast exchange takes place between different pore systems. The one exception to the trend is TiC-CDC600 with an average pore size of $0.6 \mathrm{~nm}$, for which a number of studies have shown that adsorbed 

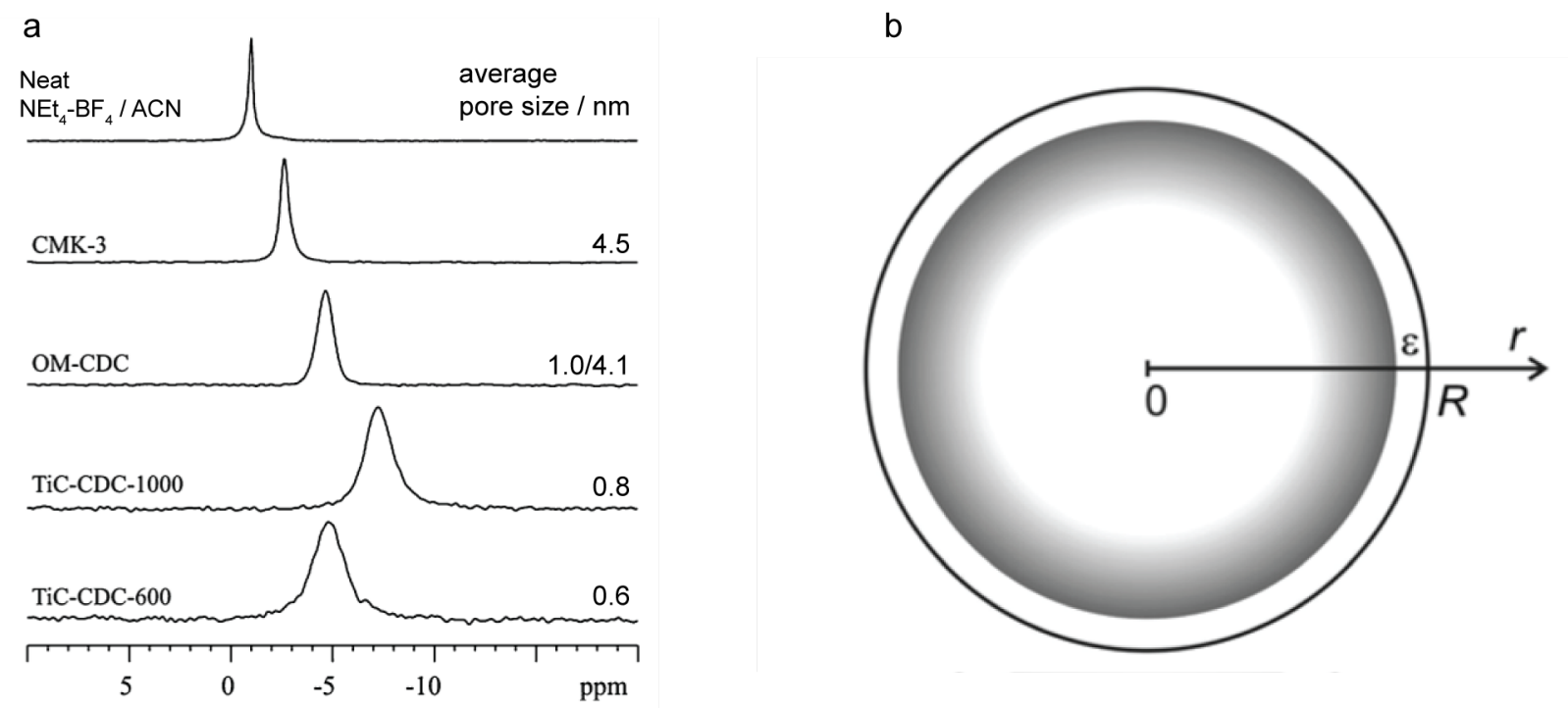

Figure 4. (a) ${ }^{11} \mathrm{~B}$ MAS NMR spectra of porous carbon materials with well defined pore-sizes loaded with $\mathrm{NEt}_{4}-\mathrm{BF}_{4} / \mathrm{ACN}^{-}$ electrolyte. Spectra are referenced relative to the neat electrolyte (top). (b) Illustration of the spherical pore model used to rationalize the variation in chemical shift with the pore radius, $R$. $\varepsilon$ denotes the minimum distance for adsorption of an electrolyte species against a pore wall, with larger ring current shifts denoted by darker grey shading. [Adapted from Ref. ${ }^{57}$ with permission from the PCCP Owner Societies].

electrolyte species exhibit smaller than expected ring current shifts. ${ }^{57},{ }^{26},{ }^{58}$ However, ${ }^{1} \mathrm{H}$ MAS NMR experiments on the unloaded carbon indicate the presence of a significant amount of hydrogen in the material. This is indicative of a more fragmented structure in which the $s p^{2}$-hybridisation is reduced by the extensive termination of bonds by hydrogen. Indeed, quenched molecular dynamics simulations predict highly disordered and discontinuous structures for CDCs produced at low chlorination temperatures. ${ }^{28}$ In such materials, the reduced electron delocalization would be expected to result in a reduced ring current shift for in-pore species.

The solvation state of adsorbed ions has also been investigated by comparing ${ }^{13} \mathrm{C}$ and ${ }^{11} \mathrm{~B}$ MAS NMR spectra for electrolyte-loaded carbons before and after evacuation of the ACN solvent. ${ }^{57}$ For a mesoporous carbon with a pore diameter of $4.5 \mathrm{~nm}$, the results show an increase in the diamagnetic ring current shift for in-pore ions after removal of the solvent. This is consistent with the in-pore species becoming closer on average to the pore walls due to loss of their solvation shells. However, this behaviour is not observed for TiC-CDC-1000 with an average pore width of $0.8 \mathrm{~nm}$, where the ring current shift for in-pore $\mathrm{BF}_{4}$ anions reduces upon solvent removal. This may indicate that in-pore species are partially desolvated in small micropores, and that solvent removal reduces the confinement of in-pore $\mathrm{BF}_{4}$ anions. It could also indicate that removal of the solvent simultaneously removes $\mathrm{BF}_{4}$ anions from the smallest micropores, although further experiments would be needed to prove this. 


\subsection{Solid-state NMR of adsorbed species as a probe of electrode structure}

While the adsorption behaviour and ring current shifts can be linked to the local environments of adsorbed species through empirical trends for a set of well-defined materials, it can be difficult to separate pore size effects from other more complex structural parameters such as the degree of structural ordering and local curvature in the microporous carbon. One way to gain fundamental insight is through density functional theory (DFT) calculations, where NMR observables can be directly related to a known structural model. For studying ring current effects, this is most commonly done through calculation of the nucleus-independent chemical shift (NICS). ${ }^{59}$ In the NICS approach, DFT calculations are performed to determine chemical shielding tensors and their isotropic values $\left(\sigma_{\text {iso }}\right)$ at arbitrary positions in space close to a $\pi$-bonded structure. The isotropic

NICS at that position, $\delta_{\text {iso }}{ }^{\text {NICS }}$ is then given by $-\left(\sigma_{\text {iso }}-\sigma_{\text {ref }}\right)$, where the reference shielding, $\sigma_{\text {ref, }}$ is zero in the NICS approach. This approach therefore provides a measure of the ring currentinduced chemical shift change that is expected at a point in space due to a nearby $s p^{2}$-hybridised carbon surface or structure. This contrasts more conventional DFT methods which calculate NMR parameters at specific nuclear sites. The advantage of the NICS approach is that it is possible to separate chemical effects on the NMR parameters (e.g., due to physisorption or structural distortions of adsorbed species) from purely magnetic effects associated with the ring currents in the nearby delocalized electron system.

The NICS approach has been used to explore ring current shielding effects on species confined within well-defined carbon nanostructures such as nanotubes ${ }^{60},{ }^{61},{ }^{62}$ and fullerenes. ${ }^{63}$ These studies show that chemical shifts of confined species can be strongly influenced by the chemical and electronic structure of the surrounding carbon surface, and are only weakly affected by chemical interaction with the carbon surface. The NICS approach has recently been applied specifically to gain insight into ring current shifts for adsorbed species within microporous carbon. ${ }^{26}$ Carbon fragments within the microporous structure were modelled by simple coronene-based units of different diameters. For the smallest unit (coronene), the calculations predict a value of $\delta_{\text {iso }}{ }^{\text {NICS }}=-$ $4.5 \mathrm{ppm}$ at $3 \AA$ from the surface, but this reduces in magnitude significantly with increasing distance, reaching values of close to $0 \mathrm{ppm}$ at $15 \AA$ from the surface (Figure $5 \mathrm{a}$ ). This shows that the ring current effect is highly dependent on the proximity to the carbon surface and confirms that significant shifts should only be observed for species residing at distances relevant to molecular adsorption. However, proximity to the carbon surface is not the only factor that influences the ring current shift. Increasing the size of the carbon domain also increases the magnitude of the NICS at a given distance from the surface by several ppm (Figures $5 b, c$ ). These results show that the magnitude of the ring current shift can also be used to probe the ordering of the carbon structure. Local curvature also has a significant effect on the magnitude of the NICS; calculations for a bowl- 
a) Distance above ring plane, z $(\AA)$

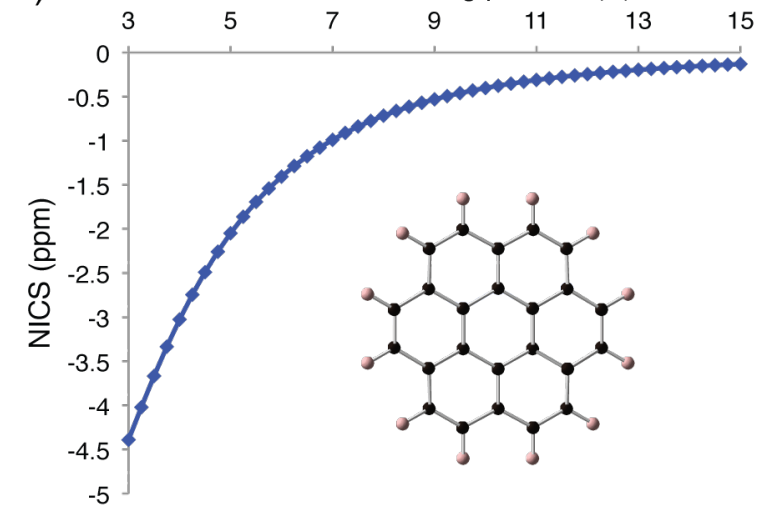

b) Distance above ring plane, $z(\AA)$

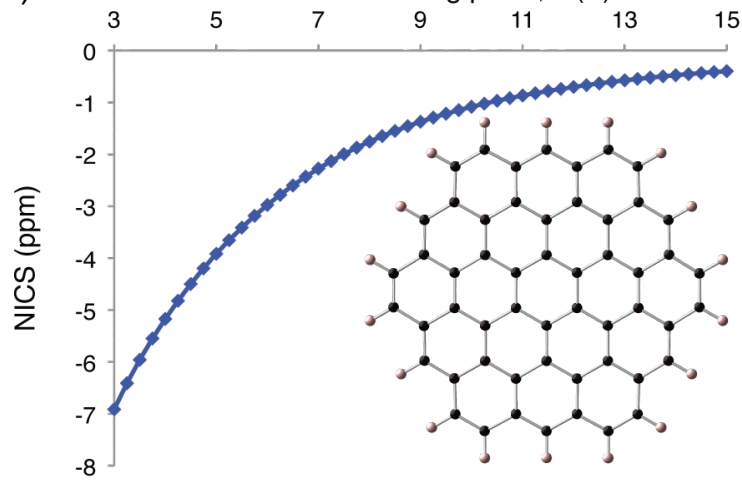

c)

Distance above ring plane, $z(\AA)$

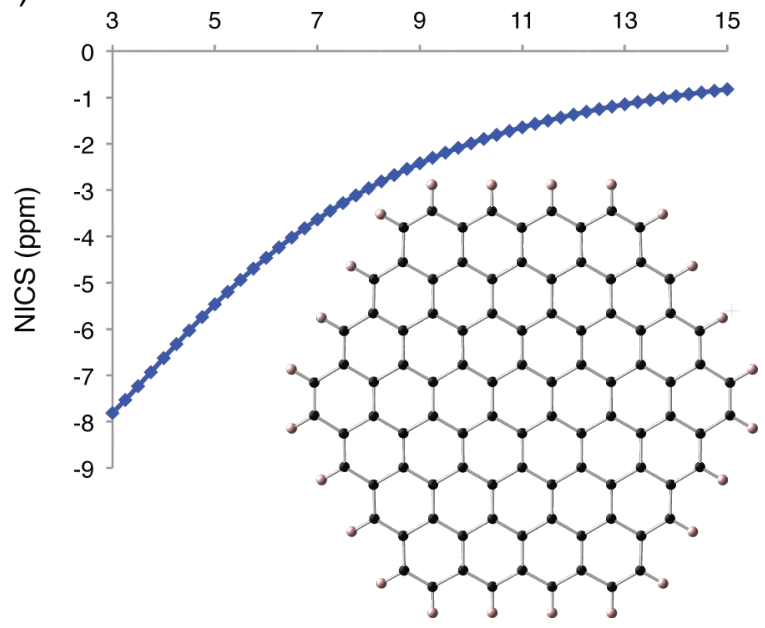

Figure 5. Calculated isotropic NICS values at distances between 3 and $15 \AA$ from the surface of (a) coronene, (b) circumcoronene and (c) dicircumcoronene model carbon fragments. Isotropic NICS values were calculated vertically above the centre of each model carbon fragment. [Adapted with permission from Ref. ${ }^{26}$. Copyright 2014 American Chemical Society].

shaped corannulene unit predict increased NICS values near the concave face, but reduced NICS values near the convex face. Pore-size effects were modelled by creating a slit pore from two parallel circumcoronene sheets. The results show that the distance dependence of the NICS within such a slit pore is additive, being essentially equal to the sum of the NICS from both surfaces. For narrow slit pores, this leads to overlap of the shielding effects from both pore walls, resulting in much larger NICS values. It is also important to account for the effects of dynamics, since in real 
carbon micropores adsorbed species are expected to undergo fast exchange between different positions within the pore, resulting in averaging of the NICS. Taking this into account, an average $\delta_{\text {iso }}{ }^{\text {NICS }}$ value of $-8.2 \mathrm{ppm}$ was determined for a $1 \mathrm{~nm}$ pore width, and a value of $-3.7 \mathrm{ppm}$ for a 2 $\mathrm{nm}$ pore width. This shows that while the ring current shift reduces with increasing pore width, measurable effects can still be expected even for species adsorbed within relatively large micropores and small mesopores.

The NICS calculations show that multiple factors are expected to contribute to the measured ring current shift. In real systems, it can be difficult to separate the different contributions without prior knowledge of the complex carbon structure. However, TiC-CDCs again provide a convenient model system for which structural properties such as the average pore size are well constrained. The insight gained from the NICS calculations has been used to interpret ring current shifts measured for adsorbed species in a set of TiC-CDCs prepared at chlorination temperatures of 600 , 800 and $1000{ }^{\circ} \mathrm{C}$, with respective average pore diameters of $0.82,0.91$ and $0.93 \mathrm{~nm} .{ }^{26}$ The magnitude of the experimental ring current shift for adsorbed $\mathrm{BF}_{4}$ anions (as measured by ${ }^{19} \mathrm{~F}$ MAS NMR) increases with increasing pore size. This trend is opposite to what is expected based on the pore-size effect; instead the results suggest that the measured ring current shift is primarily influenced by the sizes and ordering of the carbon domains within the structure. This is consistent with experimental studies based on Raman ${ }^{64}$ and X-ray spectroscopies ${ }^{29}$ and measurements of the magnetic and conductive properties, ${ }^{27}$ which indicate that more extended and continuous carbon domains are present in carbons which have been treated at higher temperatures.

NICS calculations on model slit pores provide key insights into the origin of ring current shifts, allowing experimental results for carbons with well-defined pore sizes to be interpreted in terms of the local structure of the carbon. However, many microporous carbons exhibit additional levels of structural complexity that are not captured by NICS calculations on model carbon fragments. In particular, it is difficult to fully account for the effects of a distribution of pore sizes and the dynamic exchange of electrolyte species between different pores. To study this requires structural models that are currently too large to be feasible by DFT. To address this problem, Merlet et al. have combined the NICS approach with dynamic information obtained from molecular dynamics simulations in a lattice simulation method. ${ }^{65}$ In this approach, the free energy profile for molecular adsorption on a carbon surface is obtained from molecular dynamics simulations and combined with ring current shifts determined from DFT-NICS calculations. It is then possible to account for ion dynamics within the slit pore as well as exchange dynamics between multiple pores of different widths. The inclusion of a realistic adsorption free energy profile, as opposed to assuming a flat free energy profile, led to measurable changes of the chemical shifts for species confined inside slit 
pores. Interestingly, the spatial distribution of slit-pores of different sizes within a carbon particle was shown to affect the lineshapes for confined species. The validity and utility of the lattice simulation-DFT approach has subsequently been demonstrated for the structural study of microporous carbons with different pore and domain sizes. ${ }^{58}$ Through comparison of the experimental and simulated ring current shifts for adsorbed $\mathrm{BF}_{4}$ anions, approximate domain sizes were determined in absolute terms for a set of TiC-CDCs subjected to different high-temperature heat treatments. For TiC-CDC-600, the average domain size determined in this way is smaller than the size of a coronene molecule (7.5 ̊ diameter), whereas for TiC-CDC-1000, the average domain size is intermediate between coronene and circumcoronene (12.4 $\AA$ diameter). High-temperature vacuum treatment significantly increases the average domain size to be comparable with dicircumcoronene (17.3 Å diameter). 


\section{Insight into Supercapacitor Charging Mechanisms}

\subsection{Relating in-pore resonance intensities to the charging mechanism}

The integrated intensity of the in-pore resonance provides a quantitative measure of the number of in-pore species in the supercapacitor electrode. NMR measurements on charged supercapacitor electrodes can therefore offer a unique insight into the one of the key questions regarding the fundamental mechanism of supercapacitance: how do in-pore ion populations change in order to balance the electronic charge that is developed on the electrode surface? The traditional assumption is that counter-ions (ions of opposite charge to the electrode surface) are absorbed into the micropores to balance the electronic charge that accumulates in the electrode surface. However, in reality the charge storage process is more complex and a number of different mechanisms are possible. ${ }^{43}$ Importantly, the NMR adsorption studies described in the previous section clearly show that a significant number of ions are already present inside micropores even in uncharged electrodes. This means there are a number of possible mechanisms that can lead to the build up of ionic charge inside the micropores, as illustrated in Figure 6. Counter-ions can be adsorbed to balance each unit of electronic charge on the electrode surface (Figure 6a) resulting in an overall increase in the in-pore ion population. However, it is also possible to swap in-pore coions (ions of the same charge as the electrode surface) for ex-pore counter-ions, as shown in Figure $6 \mathrm{~b}$. In this case, each ion swap balances two units of electronic charge on the electrode surface and the total population of in-pore ions does not change. A further possible mechanism is for co-ions to desorb and be expelled from the pores, leaving a surplus of in-pore counter-ions as shown in Figure 6c. In this case, one in-pore co-ion is expelled for each unit of charge on the electrode surface and the total in-pore ion population reduced. In principle it is also possible for combinations of these charging mechanisms to take place, or for different charging mechanisms to take place in different electrodes.

The different possible charging mechanisms can be distinguished through quantitative analysis of the in-pore resonance intensities. An increase in the in-pore resonance intensity during charging shows that the studied ions are adsorbed into the micropores, whereas a decrease indicates ion expulsion. A constant in-pore resonance intensity during charging shows that the population of inpore ions under study remains constant, and would imply that the population of the other type of ionic species must be changing (either through adsorption or desorption) in order to balance the charge on the electrode surface. By studying both ions simultaneously (by recording e.g., ${ }^{13} \mathrm{C}$ NMR spectra to observe $\mathrm{NEt}_{4}$ cations and ${ }^{11} \mathrm{~B} N \mathrm{NMR}$ spectra to observe $\mathrm{BF}_{4}$ anions) it is possible to construct a full picture of the charging mechanism and to determine the total ionic charge inside the micropores. By studying nuclei present in the solvent species it is also possible to observe 


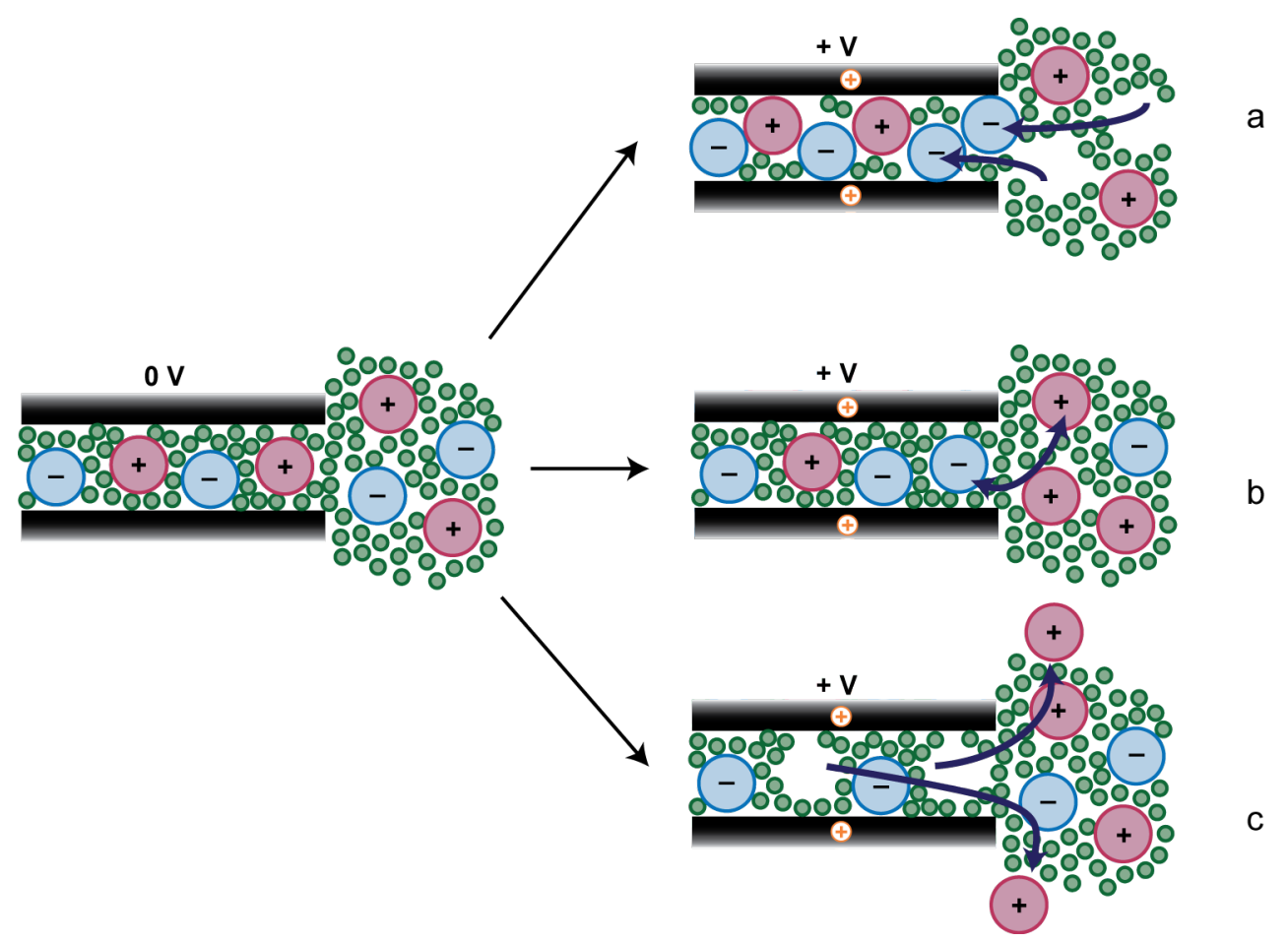

Figure 6. Schematic illustrations of possible charge storage mechanisms within a micropore that contains anions and cations prior to charging. If the electrode surface is positively polarised, an equal negative ionic charge can arise through either (a) adsorption of ex-pore anions into the pores, (b) exchange of ex-pore cations for in-pore anions or (c) the expulsion of cations from the pores. [Reproduced from Ref. ${ }^{43}$ with permission from The Royal Society of Chemistry.].

changes in the in-pore solvent population which can then be correlated with the charging mechanism determined by the in-pore ion populations.

\subsection{In-pore chemical shift changes during charging}

In addition to changes in the in-pore resonance intensity, NMR spectra of supercapacitor electrodes generally exhibit a second phenomenon whereby the in-pore resonance is observed to move to higher chemical shift by several ppm during charging. While it may appear counterintuitive, this effect is not primarily due to changes in the proximity of the ions to the carbon surface. Indeed, the proximity to the carbon surface does affect the magnitude of the ring current shift, yet DFT calculations have shown that small displacements relevant the movement of ions closer to or further from the carbon surface within a micropore cannot account for the magnitudes of the chemical shift changes that are typically observed. ${ }^{66}$ Furthermore, the in-pore resonance frequency changes are generally in the same direction regardless of polarization of the electrode surface or the nucleus studied, and are approximately equal for anions, cations and even solvent species. Instead, this phenomenon is due to the changes in the electronic structure of the carbon surface as the electrode is charged. As explained in Section 3, the exact magnitude and sign of the ring current shift depend precisely on the electronic structure of the carbon. During charging, electrons or holes accumulate on the electrode surface and are balanced by the ionic charge developed in the electrolyte. The unpaired electrons and holes result in an increasingly 

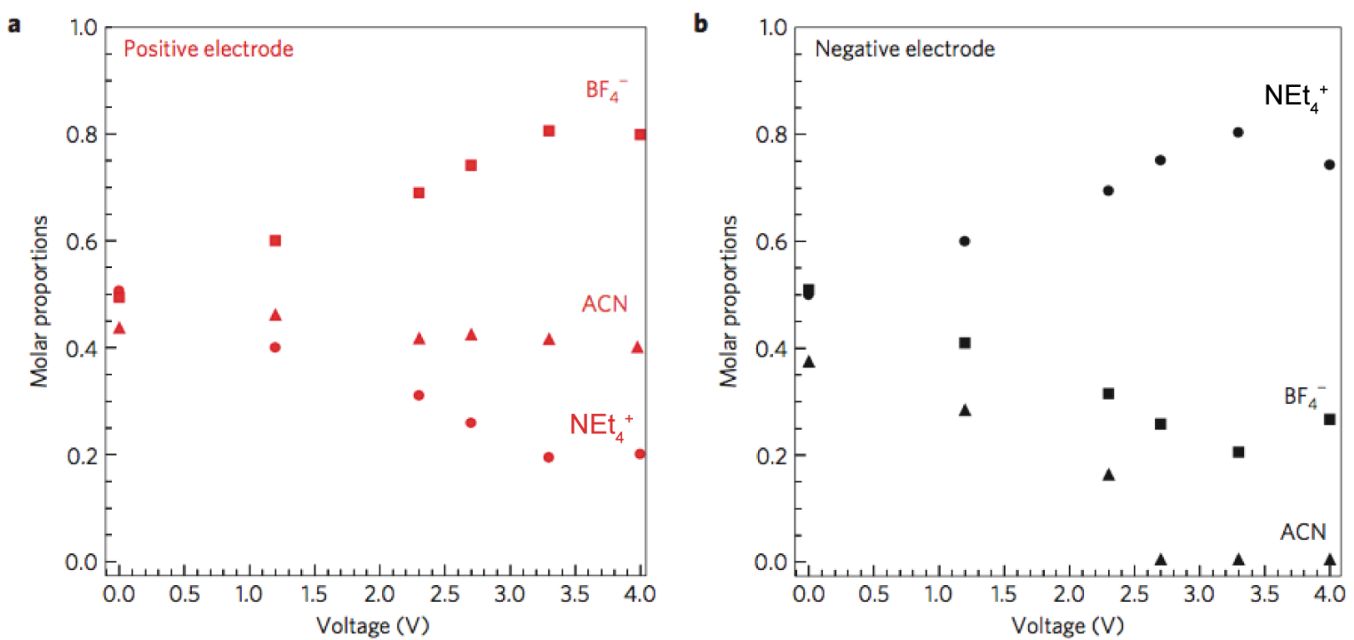

Figure 7. Molar proportions of in-pore $\mathrm{NEt}_{4}-\mathrm{BF}_{4} / \mathrm{ACN}$ electrolyte species determined from ex situ NMR experiments on charged electrodes. [Figure reproduced with permission from Ref. ${ }^{41}$. Reprinted by permission from Macmillan Publishers Ltd.: Nature Materials, copyright (2013)].

paramagnetic contribution to the ring current as the electrode is charged, causing resonances species near the carbon surface to move higher chemical shift in the NMR spectrum. This has been qualitatively confirmed by DFT calculations which show that the ring current associated with a small carbon fragment changes sign from diamagnetic to strongly paramagnetic when either a positive or negative charge is applied. This mirrors experimental observations for [18]annulene, where the ${ }^{1} \mathrm{H}$ chemical shifts of the interior protons changed from $-3.0 \mathrm{ppm}$ to 28.1 and $29.5 \mathrm{ppm}$ following two electron reduction. ${ }^{67}$ The change in chemical shift observed for in-pore species therefore does not provide direct information about the behaviour and rearrangment of ions inside the micropores, but instead can be related to the amount and distribution of the electronic charge on the carbon surface.

\subsection{Ex situ solid-state NMR methods for studying supercapacitor electrodes}

The selective yet quantitative nature of solid-state NMR makes it an ideal probe to study in-pore ion populations in supercapacitor electrodes, which can then be used to distinguish between the different possible charging mechanisms. However, unlike adsorption studies where samples can be prepared by pre-soaking the microporous carbon sample with electrolyte, NMR experiments to probe the charging mechanism must be performed on charged electrodes. One way to do this is by ex situ NMR, where supercapacitor test cells (e.g., coin cells) are charged to a specified voltage, and then disassembled to obtain the electrode for study by NMR. The advantage of ex situ NMR is that the electrode can be studied by conventional MAS techniques, which provide high resolution and in principle enable complex experiments such as correlations and exchange experiments. However, ex situ NMR also involves practical challenges; the extraction of the electrodes from charged supercapacitor cells must done very carefully in an inert environment (inside a glove box) 
to avoid discharging the capacitor or introducing water into the sample, and evaporation of the solvent during cell disassembly can result in solidification of the electrolyte salt, potentially altering the local environments of the in-pore species.

Ex situ MAS NMR has been used to study the behaviour of $\mathrm{NEt}_{4}-\mathrm{BF}_{4} / \mathrm{ACN}$ electrolyte ions and solvent molecules in activated carbon supercapacitor electrodes. ${ }^{41}$ For uncharged electrodes, ${ }^{13} \mathrm{C}$ MAS NMR spectra reveal two distinct environments for the in-pore $\mathrm{NEt}_{4}$ cations and $\mathrm{ACN}$ solvent molecules. Two-dimensional exchange experiments show that species in both adsorption sites undergo millisecond-timescale exchange with the free electrolyte, but do not exchange with each other. The exact nature of the two adsorption sites was not conclusively identified from these observations but may be related to structural heterogeneity of the activated carbon electrode materials resulting in a range of different adsorption sites. For electrodes extracted from charged cells, ${ }^{13} \mathrm{C}$ and ${ }^{11} \mathrm{~B}$ MAS NMR spectra show clear changes in the intensities of the in-pore resonances, thereby providing an insight into how the in-pore ion populations change during charging (Figure 7). In positively polarized electrodes, the in-pore $\mathrm{BF}_{4}$ anion population increases, while the in-pore $\mathrm{NEt}_{4}$ cation population decreases; in the negative electrode, the opposite behaviour is observed. These observations are consistent with an ion swapping charging mechanism whereby co-ions are expelled from the pores while counter-ions are simultaneously absorbed. In addition to following changes in the ionic populations within the micropores, ${ }^{13} \mathrm{C}$ MAS NMR was also used to observe changes in the in-pore solvent molecule populations. Interestingly, these show different behaviour in positively- and negatively-polarised electrodes; the in-pore solvent population remains approximately constant under positive polarisation, whereas it decreases under negative polarisation. This could be taken to indicate ion desolvation in the negative electrode; however, the drying procedure that was employed prior to the NMR measurement in this study may have also affected the in-pore solvent population.

${ }^{19} \mathrm{~F}$ and ${ }^{1} \mathrm{H}$ ex situ MAS NMR experiments have been used to study the factors affecting ionic mobility and the charge storage mechanism in supercapacitors containing the RTIL electrolytes 1methyl-1-propylpyrrolidinium bis(trifluoromethanesulfonyl)imide ( $\mathrm{Pyr}_{13}$-TFSI), and 1-ethyl-3methylimidazolium bis(trifluoromethanesulfonyl)imide (EMI-TFSI). ${ }^{68}$ As mentioned in the introduction, while RTIL electrolytes can increase the energy stored due to their higher breakdown voltages, one of the key drawbacks is their increased viscosity and lower power capabilities. The increased viscosity of RTILs also presents challenges from the point of view of NMR spectroscopy; indeed for both electrolytes, the MAS NMR spectra show substantial broadening of the in-pore resonances due to the lower ionic mobility within the micropores. Despite this, the observation of in-pore electrolyte species in uncharged electrodes demonstrates unambiguously that the ionic 


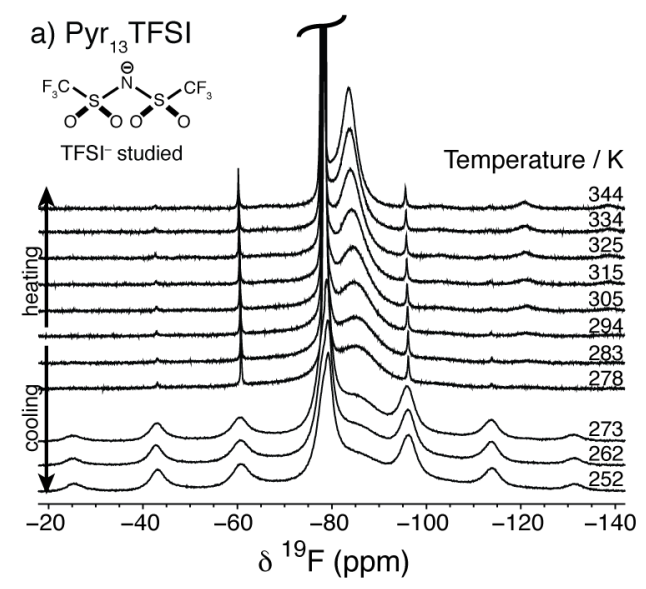

c) $\mathrm{TFSI}^{-}$
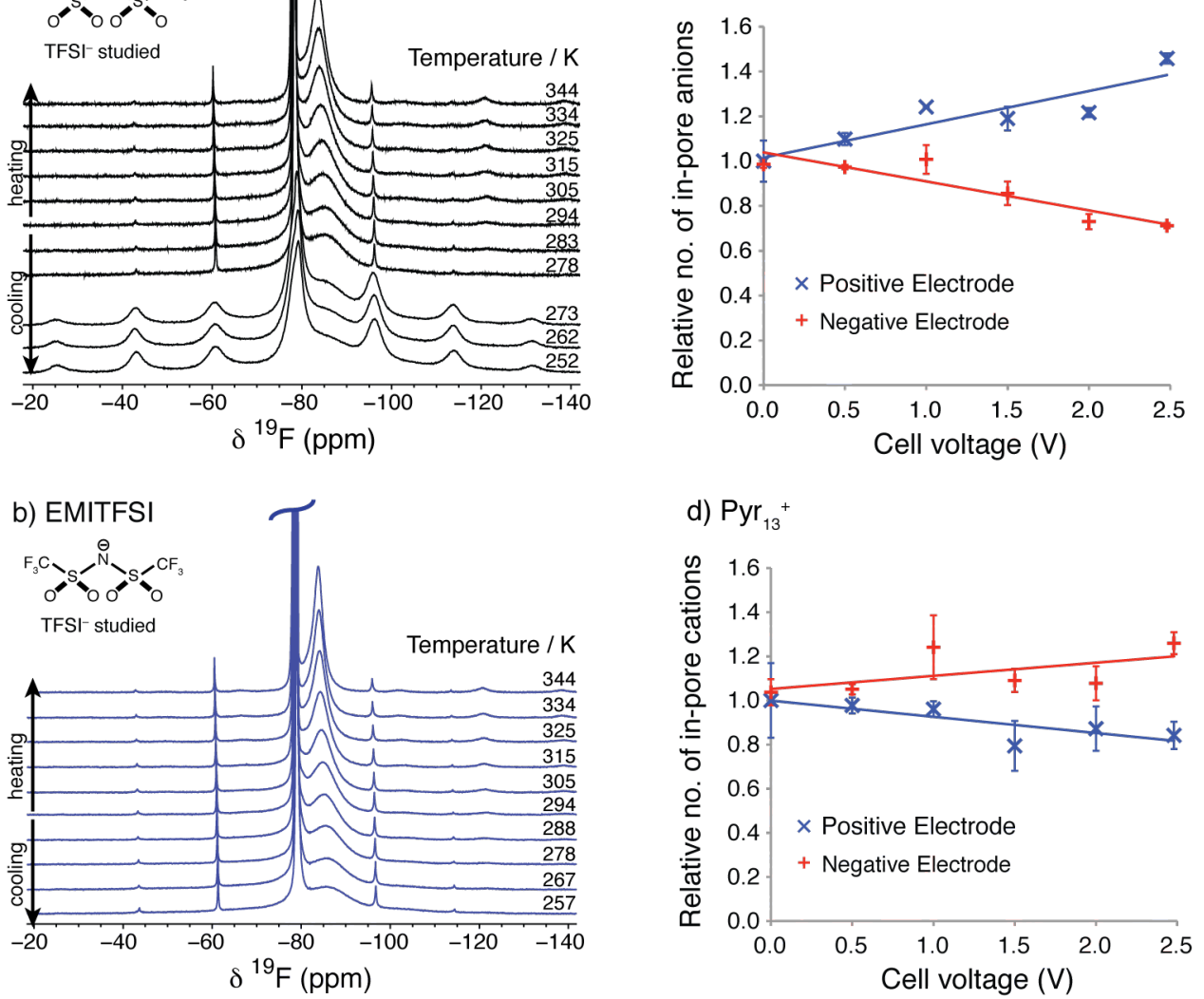

Figure 8. Variable-temperature ${ }^{19} \mathrm{~F}$ MAS NMR spectra of YP-50F activated carbon supercapacitor electrodes loaded with (a) $\mathrm{Pyr}_{13}$-TFSI and (b) EMI-TFSI RTIL electrolytes. Relative populations of in-pore (c) TFSI anions and (d) Pyr ${ }_{13}$ cations in electrodes taken from dissembled supercapacitor are shown as a function of the cell voltage. [Adapted with permission from Ref. ${ }^{68}$. Copyright 2015 American Chemical Society].

liquids spontaneously wet the pores in the absence of an applied potential, in agreement with molecular dynamics simulations. ${ }^{36}$ However, through deconvolution and integration of the in-pore resonance, the results show that only $40 \%$ of the total pore volume (as measured by $\mathrm{N}_{2}$ sorption) is accessed by the electrolyte ions, indicating that RTILs are not able to fully penetrate the porous network. Variable-temperature ${ }^{19} \mathrm{~F}$ NMR spectra (Figures $\left.8 a, b\right)$ show significant narrowing of the in-pore resonances for both electrolytes with increasing temperature. This can be interpreted in terms of increased exchange rate of the TFSI anions between different adsorption sites within the porous network. At the maximum temperature of $344 \mathrm{~K}$, no motional broadening of the ex-pore resonances is observed, confirming that exchange between the in-pore and ex-pore environments takes place on a much slower timescale. The in-pore linewidths are consistently narrower for the EMI-TFSI sample, showing that the in-pore TFSI anions are much more mobile in this RTIL. This helps to explain the better power performance of supercapacitors using EMI-TFSI as opposed to Pry $_{13}$-TFSI. Further experiments showed that the addition of ACN solvent to the electrolyte reduces 
the in-pore ion population and significantly increases the mobility of in-pore ions as evidenced by a reduction in the in-pore linewidth. This is also accompanied by slight increases in the fraction of the microporous carbon network accessed by the electrolytes, although this volume is now shared between RTIL and solvent molecules. The faster in-pore ionic motion following the addition of ACN helps to explain the observed improvement of power performance in supercapacitor cells.

Quantitative insight into the charge storage mechanism was obtained by studying electrodes extracted from charged $\mathrm{Pyr}_{13}$-TFSI supercapacitors. Deconvoluted in-pore resonance intensities in ${ }^{1} \mathrm{H}$ and ${ }^{19} \mathrm{~F}$ MAS NMR spectra enabled the relative proportions of in-pore anions and cations to be quantified independently (Figures $8 \mathrm{c}, \mathrm{d}$ ). Similar to the $\mathrm{NEt}_{4}-\mathrm{BF}_{4} / \mathrm{ACN}$ electrolyte studied previously, ion swapping is the predominant mechanism for both positive and negative charging. However, the relative change in the in-pore anion population is larger than that of the in-pore cation population, meaning there is a small net adsorption of anions and therefore an increase in the total population of in-pore ions in the positive electrode. The additional volume required to accommodate these ions may come from the electrolyte accessing areas of the microporous network that were previously not wetted before charging, or through ordering of the ions within the electrolyte species. Indeed, recent X-ray diffraction experiments have indicated vertical ordering of solvent molecules in small micropores during charging. ${ }^{69}$

\subsection{In situ NMR of supercapacitors: Experimental considerations}

Some of the practical issues associated with ex situ NMR can be addressed by performing in situ NMR experiments on working devices. This approach is very similar to in situ NMR of batteries, ${ }^{70}$ whereby a specially-designed supercapacitor test cell is placed inside the coil of a static NMR probe and connected to an external electrochemical cycler. In situ NMR has the advantage that the supercapacitor can be studied directly under a range of charging conditions with no risk of discharge or loss of solvent during cell disassembly. In principle this should provide a more accurate picture since potentially short-lived and/or dynamic states within the electrode can be studied without disturbing them during cell disassembly. The first in situ NMR study of supercapacitors was carried out by Wang et al., who used ${ }^{11} \mathrm{~B}$ NMR to observe changes in the environments of $\mathrm{BF}_{4}$ anions in a supercapacitor comprising $\mathrm{NEt}_{4}-\mathrm{BF}_{4} / \mathrm{ACN}$ electrolyte and activated carbon electrodes. ${ }^{71}$ This study highlighted the importance of the supercapacitor cell design for in situ NMR experiments. Unlike in situ NMR studies of batteries, where the two electrodes generally comprise different materials and can be easily distinguished in the NMR spectrum, supercapacitor electrodes are essentially chemically identical and the in-pore resonances associated with the anode and the cathode are therefore unresolved. Wang et al. introduced a solution to this problem with a 'shifted' bag cell design (Figures 9a,b). ${ }^{71}$ In this design, 
a)

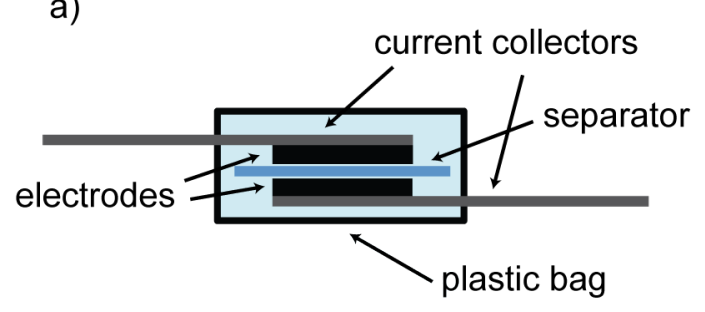

b)

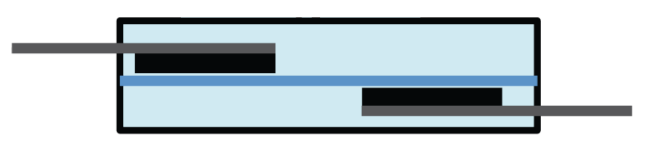

c)

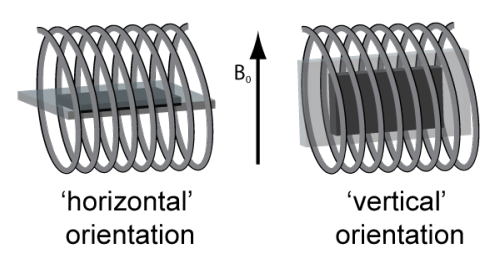

d)

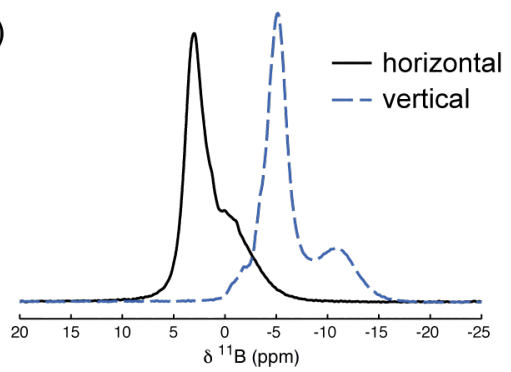

Figure 9. Schematic representations of in situ supercapacitor bag cells with (a) conventional and (b) 'shifted' designs. [Adapted with permission from Ref. ${ }^{71}$. Copyright 2011 American Chemical Society]. Horizontal and vertical cell orientations are shown in (c), with the corresponding NMR spectra shown in (d). [Reprinted from Ref. ${ }^{56}$ with permission from the PCCP Owner Societies].

the cell is constructed from flat pieces of electrode film pressed onto a mesh current collector. A small strip of porous polymer membrane separator is placed between the two electrodes and the whole assembly is heated-sealed inside a plastic bag. Before making the final seal, electrolyte is added to the supercapacitor using a microsyringe. Importantly, in the shifted-cell design, the two electrodes are displaced laterally so that a single electrode can be placed within the NMR coil. Using this setup, it was possible to study changes in the total $\mathrm{BF}_{4}$ anion populations in a single electrode under positive and negative polarization between cell voltages of $\pm 2 \mathrm{~V}$. Comparison of the total spectral integrated intensities revealed an overall decrease in the $\mathrm{BF}_{4}$ anion population for negative electrode polarisation, and an overall increase for positive electrode polarization. This qualitatively agrees with the picture from ex situ NMR whereby co-ions are desorbed and counterions are adsorbed during charging. ${ }^{41}$

Since the initial demonstration of in situ NMR for the study of supercapacitors, the approach has been further developed and optimized by taking into account various aspects of the cell design and experimental methodology. One important consideration in in situ NMR of supercapacitors is the reduction in resolution as compared to ex situ MAS NMR, since experiments are necessarily performed under static conditions. Furthermore, for highly anisotropic sample geometries such as the supercapacitor bag cells typically used, the shifts and lineshapes of the observed resonances can be strongly affected by bulk magnetic susceptibility (BMS) effects which depend upon the shape and orientation of the sample. However, for the study of supercapacitor electrodes, these BMS effects can actually be exploited to increase the resolution. Indeed, Forse et al. have shown that for bag cells containing flat rectangular electrodes, BMS effects for ex-pore and in-pore species have slightly different orientation dependences with respect to the applied magnetic field. ${ }^{56}$ 
For vertical cell orientations, the shift difference between ex-pore and in-pore resonances is larger than for horizontal orientations (as illustrated in Figures 9c,d). This means for the study of flat supercapacitor bag cells, resolution can be maximised by performing in situ NMR experiments with the cell oriented vertically.

Other improvements have also been made to the cell design. While the shifted cell allows in situ NMR spectra to be obtained independently for the two electrodes, it also significantly increases resistance within the device leading to reduced electrochemical performance, particularly at high charge and discharge rates. This problem has been addressed by 'overlaying' the current collectors in order to maximize the electric field near the electrodes. ${ }^{66}$ Direct electrical contact between the electrodes is still prevented by positioning the separator over and under the electrodes on either side of the device. The 'overlaid' design gives improved electrochemical performance in cyclic voltammetry experiments where the cell voltage is swept across a defined range at a specified rate. For the 'overlaid' design, much higher currents are obtained for the same voltage sweep rate, indicating reduced resistance within the cell.

In addition to dealing with the practical issues associated with in situ NMR of supercapacitors, the choice of experimental NMR methodology can also affect the information that is obtained. While initial studies of the $\mathrm{NEt}_{4}-\mathrm{BF}_{4} / \mathrm{ACN}$ electrolyte system focused on ${ }^{11} \mathrm{~B} N M R$ for the study of the $\mathrm{BF}_{4}$ anions, Wang et al. have shown that ${ }^{19} \mathrm{~F}$ NMR can actually be a better choice. ${ }^{66}$ Owing to its higher gyromagnetic ratio and natural abundance, ${ }^{19} \mathrm{~F}$ is an intrinsically more sensitive nucleus than ${ }^{11} \mathrm{~B}$. Furthermore, for the study of $\mathrm{BF}_{4}$ anions, the molar fraction of fluorine atoms is a factor of four higher than that of ${ }^{11} \mathrm{~B}$, meaning the relative sensitivity is further increased. Together, these factors significantly reduce the experimental time meaning that in situ NMR spectra can be acquired in a matter of minutes. The main drawback associated with ${ }^{19} \mathrm{~F}$ in situ NMR of supercapacitor electrodes is that there can be a significant background signal both within the sample from the PTFE binder used in the electrode film, and externally from commonly-used PTFE probehead components. However, since these contributions typically have much shorter $T_{2}$ relaxation times, and/or different nutation behaviour, they can be effectively removed through the use of the 'depth' background suppression pulse sequence. ${ }^{72}$

\subsection{In situ NMR of supercapacitors: Insight into the charging mechanism}

Combining the optimized cell design and experimental methodology, Wang et al. performed a more detailed in situ NMR study of a supercapacitor comprising activated carbon electrodes and $\mathrm{NEt}_{4}$ $\mathrm{BF}_{4} / \mathrm{ACN}$ electrolyte. ${ }^{66}$ Changes in the in-pore anion populations during charging were monitored by recording ${ }^{19} \mathrm{~F}$ in situ NMR spectra with the cell held at fixed voltages. The results show that for 
negative electrode polarization, the in-pore anion population decreases slightly upon charging to $0.5 \mathrm{~V}$, after which larger decreases are observed up to $-1.25 \mathrm{~V}$. For positive electrode polarization, the in-pore anion population remains approximately constant between $0-0.75 \mathrm{~V}$ before increasing. From this it can be indirectly inferred that charge storage at low polarisations is achieved through adsorption/desorption of the $\mathrm{NEt}_{4}$ cations, since the in-pore $\mathrm{BF}_{4}$ anion population remains approximately constant. For larger polarisations adsorption and desorption of the $\mathrm{BF}_{4}$ anions from the micropores starts to play a more important role in charge storage. This transition between different charging regimes was not previously observed by ex situ MAS NMR. However, the observations are consistent with other work based on in situ electrochemical quartz-crystal microbalance (EQCM) measurements on a similar system, which indicate anion-cation mixing inside the micropores at low electrode polarisations, and increasing contributions from counter-ion adsorption at high electrode polarisations. ${ }^{73}$

In addition to the study of in-pore anion populations at fixed voltages, Wang et al. also demonstrated an additional in situ NMR approach whereby spectra are recorded for a supercapacitor cell undergoing simultaneous cyclic voltammetry. ${ }^{66}$ This approach provides insight into the behaviour of ions under dynamic charging conditions where the cell voltage is constantly changing. Since the duration of each ${ }^{19} \mathrm{~F}$ in situ NMR experiment was very short $(2 \%$ of the duration of the whole voltage cycle), each spectrum can be considered as a 'snapshot' at that particular point in the cycle. Although no longer fully quantitative due to the reduced recycle interval used, the behaviour of the in-pore resonance under dynamic charging conditions is qualitatively similar to that at fixed voltages; both positive and negative electrode polarisations result in movement of the resonance to higher chemical shift and changes in the resonance intensity. Stacking the consecutive NMR spectra over multiple cycles to form a pseudo-2D plot (Figure 10) reveals this behaviour to be highly repeatable and perfectly synchronized with the applied cell voltage. However, charging at higher rates results in a slight lag between the chemical shift and the cell voltage. This lag corresponds exactly to the lag between the voltage cycle and the direction of the current flow within the device, confirming that the chemical shift of the in-pore resonance reflects the charge state of the carbon surface, and not the applied cell voltage. The ${ }^{19} \mathrm{~F}$ in situ NMR approach is not limited to the study of the $\mathrm{NEt}_{4}-\mathrm{BF}_{4} / \mathrm{ACN}$ electrolyte.

Griffin et al. have studied supercapacitors comprising activated carbon electrodes and a range of fluorine-containing electrolytes in order to gain insight into the effects of relative cation/anion sizes. ${ }^{43}$ For a tetrabutylammonium $\left(\mathrm{NBu}_{4}\right)-\mathrm{BF}_{4} / \mathrm{ACN}$ electrolyte, the results show a significant decrease in the in-pore anion population during negative electrode polarization, contrasting the $\mathrm{NEt}_{4}-\mathrm{BF}_{4} / \mathrm{ACN}$ electrolyte where smaller changes have been observed, especially for low 

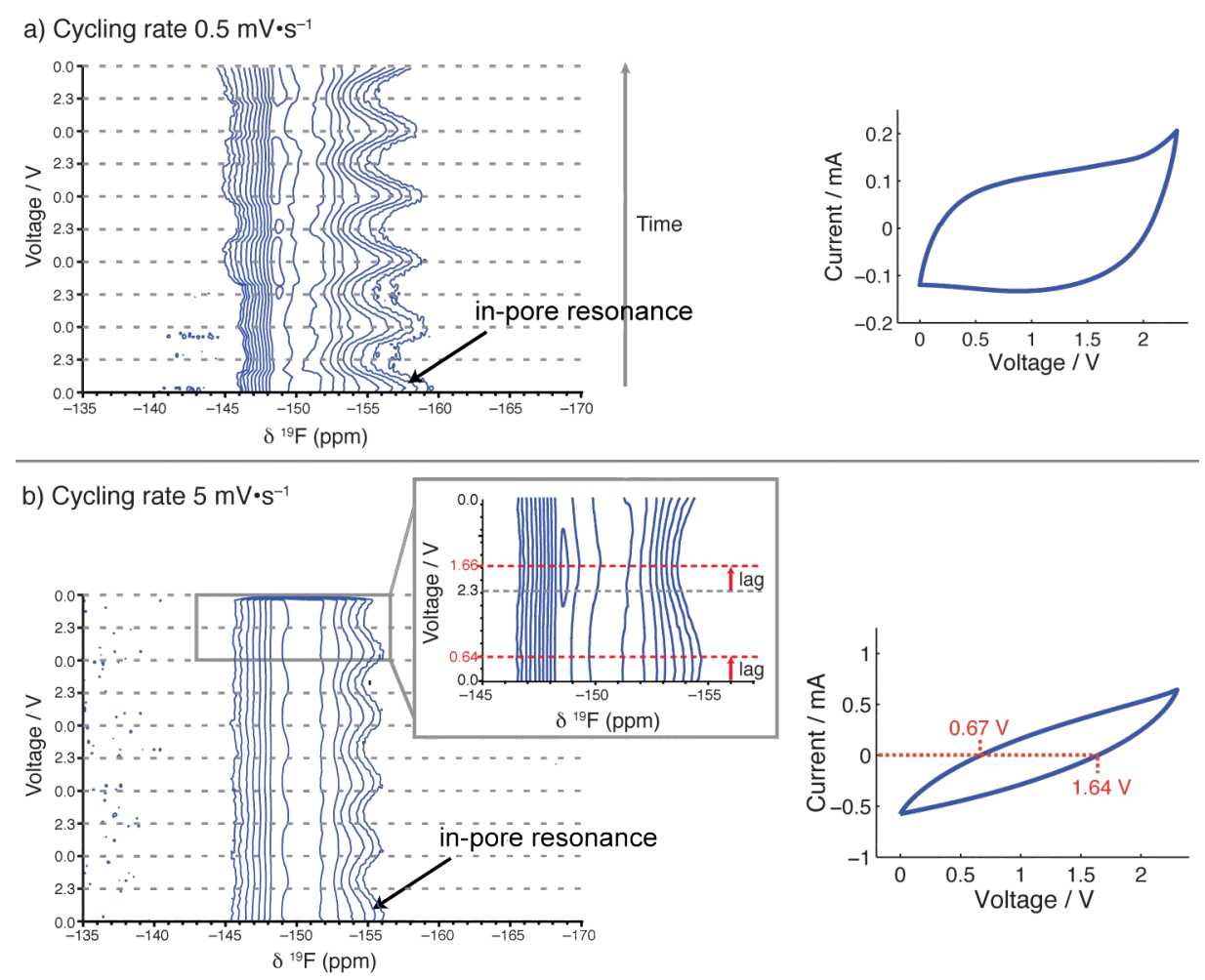

Figure 10. Contour plots showing stacked ${ }^{19} \mathrm{~F}$ in situ NMR spectra for a YP-50F activated carbon supercapacitor with $\mathrm{NEt}_{4}-\mathrm{BF}_{4} / \mathrm{ACN}$ electrolyte undergoing cyclic voltammetry at rates of (a) 0.5 and (b) $5 \mathrm{mV} \cdot \mathrm{s}^{-1}$. For the lower cycling rate, the oscillation of the in-pore resonance between high and low chemical shift is synchronized with the cell voltage; for the high cycling rate a lag is observed. [Adapted with permission from Ref. ${ }^{66}$. Copyright 2013 American Chemical Society].

electrode polarisations. This indicates that the larger size of the $\mathrm{NBu}_{4}$ cation (with an estimated desolvated diameter of $1.1 \mathrm{~nm}$ ) means it cannot access a larger fraction of the micropores, and so the $\mathrm{BF}_{4}$ anions are forced to contribute more to the charge storage mechanism. Interestingly, LiTFSI / ACN and Na-TFSI / ACN electrolytes also show large changes in the in-pore anion populations for both positive and negative polarization - as was also observed for RTILs with the TFSI anions. ${ }^{68}$ Unlike the tetraalkylammonium electrolytes, in the TFSI-based electrolytes the anion is significantly bigger than the cation (although the cations may be strongly solvated thereby increasing their effective radius). These results indicate that other factors such as the ionic charge density and ion-carbon interaction energies may also play a role in determining the charging mechanism.

While the ${ }^{19} \mathrm{~F}$ in situ NMR approach provides quantitative insight into the in-pore anion population during charging of supercapacitor electrodes, to obtain a full picture of the charging mechanism it is also necessary to quantify the in-pore cation population under the same conditions. Unfortunately, this proves to be a significant challenge due to the nature of the NMR-active species present in common electrolyte cations. For the $\mathrm{NEt}_{4}-\mathrm{BF}_{4} / \mathrm{ACN}$ electrolyte, the study of ${ }^{14 / 15} \mathrm{~N}$ or ${ }^{13} \mathrm{C}$ NMR is precluded owing to the low sensitivity of these nuclei. The most feasible nucleus is ${ }^{1} \mathrm{H}$; however, ${ }^{1} \mathrm{H}$ in situ NMR spectra are complicated by the fact that there are two chemically-distinct 
postive polarisation

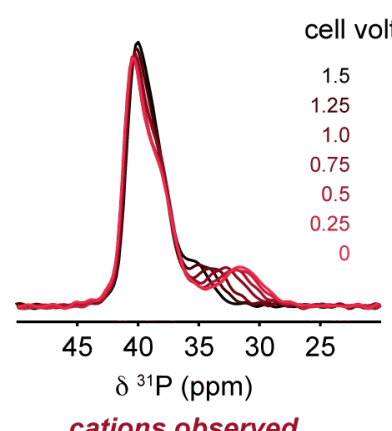

cations observed b

negative polarisation
C

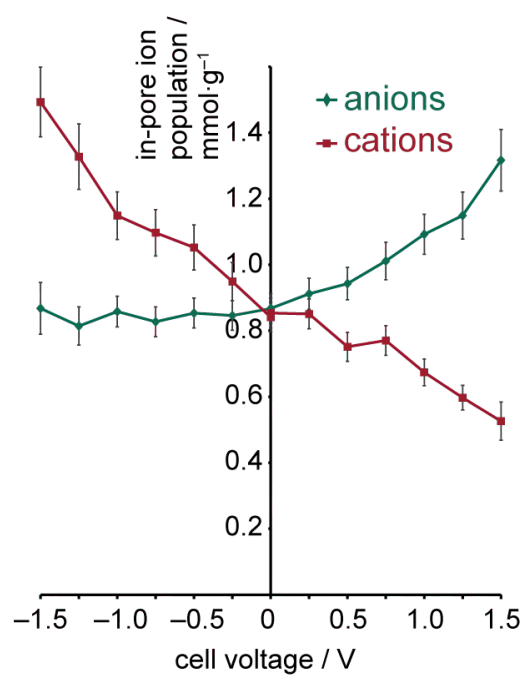

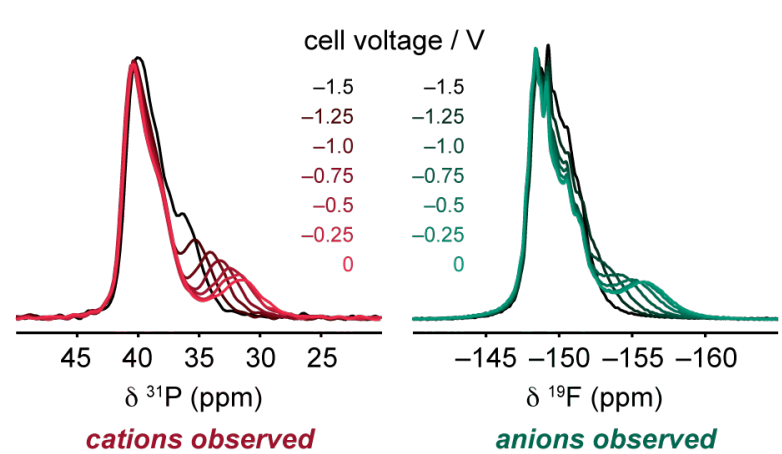

d

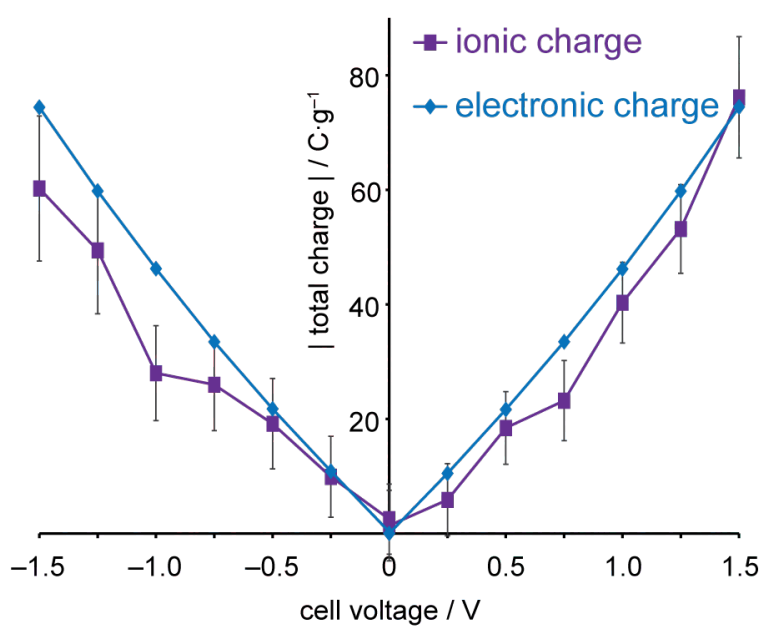

Figure 11. $(\mathrm{a}, \mathrm{b}){ }^{31} \mathrm{P}$ and ${ }^{19} \mathrm{~F}$ in situ NMR spectra of a supercapacitor containing $\mathrm{PEt}_{4}-\mathrm{BF}_{4} / \mathrm{ACN}$ electrolyte. (c) In-pore ion populations as a function of cell voltage revealing an ion exchange mechanism for positive electrode polarization and counter-ion adsorption for negative electrode polarization. (d) A comparison of the total ionic and electronic charge in the electrode as a function of cell voltage, showing that the two quantities are approximately equal. [Figure reproduced with permission from Ref. ${ }^{74}$. Reprinted by permission from Macmillan Publishers Ltd.: Nature Materials, copyright (2015)].

${ }^{1} \mathrm{H}$ environments (the $\mathrm{CH}_{3}$ and $\mathrm{CH}_{2}$ groups in the ethyl chains), and also because the polymer bag cell contributes an intense background signal to the NMR spectrum which cannot be removed even through the use of background suppression pulse sequences. This problem was recently circumvented by Griffin et al. who substituted $\mathrm{NEt}_{4}-\mathrm{BF}_{4}$ for a very similar salt, tetraethylphosphonium $\left(\mathrm{PEt}_{4}\right)-\mathrm{BF}_{4}{ }^{74}$ By choosing a cation containing phosphorous instead of nitrogen, the relatively high sensitivity ${ }^{31} \mathrm{P}$ nucleus can be studied by in situ NMR in order to monitor the in-pore cation population, while ${ }^{19} \mathrm{~F}$ in situ NMR can still be used to observe the in-pore anions. Using supercapacitors comprising activated carbon electrodes and three $\mathrm{PEt}_{4}-\mathrm{BF}_{4} / \mathrm{ACN}$ electrolytes with different concentrations, in situ NMR spectra of both the cations and anions were recorded at fixed cell voltages between $\pm 1.5 \mathrm{~V}$ (Figures 11a,b). The in-pore ion populations were quantified in absolute terms through comparison of the integrated in-pore resonance intensities with reference spectra recorded for samples with known volumes of electrolyte (Figure 11c). At 0 $\mathrm{V}$, the results show approximately equal in-pore anion and cation populations for concentrations of 
$0.5 \mathrm{M}, 0.75 \mathrm{M}$ and $1.5 \mathrm{M}$, confirming that electroneutrality is preserved inside the micropores in uncharged electrodes. For the highest electrolyte concentration, the total electrolyte ion volume estimated on the basis of the solvated ionic radii slightly exceeds the total pore volume as measured by gas sorption. This indicates that even at $0 \mathrm{~V}$ the ions in high concentration electrolytes are densely packed within the micropores, with partial desolvation or overlap of the solvation shells. During charging under fixed voltage conditions, the combined ${ }^{31} \mathrm{P} /{ }^{19} \mathrm{~F}$ in situ NMR approach reveals different charging mechanisms for positive and negative electrode polarizations. For positive polarization, charging proceeds by exchange of the in-pore cations for anions, whereas for negative polarization, cation adsorption is the dominant mechanism and the population of in-pore anions remains approximately constant. This behaviour is independent of the electrolyte concentration, indicating that other factors such as the asymmetry in ion sizes/shapes, charge density and screening, and possible differences in ionic mobility may affect the charging mechanism. Importantly, the total ionic charge, as determined from the sum of the in-pore anion and cation populations, was found to be in good agreement with the electronic charge stored in the supercapacitor (Figure 11d), confirming that the charge stored on the electrode surface is balanced by the charge associated with the ionic species inside the micropores.

In addition to experiments on 'shifted' cells, other cell designs and in situ experimental NMR methodologies have also been developed. Luo et al. developed an ingenious method whereby a copper shield surrounds one of the supercapacitor electrodes. ${ }^{75}$ Radiofrequency radiation cannot reach the shielded electrode, and the other (unshielded) other electrode is then studied in the NMR experiments. This approach was recently used to study supercapacitors with activated carbon electrodes and aqueous electrolytes. ${ }^{76}$ First, an activated carbon with relatively small pores (average width $0.58 \mathrm{~nm}$ ) was studied with the electrolyte $\mathrm{Na}-\mathrm{F}\left(0.8 \mathrm{~mol} \mathrm{~kg}{ }^{-1}\right)$. Interestingly no inpore ion resonances were detected in the absence of an applied potential, despite the presence of in-pore water. This shows that ions were unable to access the small micropores of the studied material in the absence of an applied potential. Further experiments on a second carbon material with larger pores (average width $1.58 \mathrm{~nm}$ ) revealed in-pore ion resonances at $0 \mathrm{~V}$, confirming that the lack of in-pore ions in the small pore carbon is due to a steric effect. Charging experiments on supercapacitors with the small pore-size carbon revealed that $\mathrm{F}^{-}$and $\mathrm{Na}^{+}$ions were able to enter the electrode pores when voltages of above $\sim 0.4 \mathrm{~V}$ were applied. Charging then occurred by counter-ion adsorption. These findings suggest that the electrostatic interactions between the ions and the carbon surface can overcome the steric effects associated with the small carbon pores. Interestingly, a large (negative) in-pore chemical shift change was observed in the ${ }^{19} \mathrm{~F}$ NMR spectra at voltages above $\sim 0.8 \mathrm{~V}$. This was explained by suggesting the $\mathrm{F}^{-}$ions are dehydrated at these potentials. 

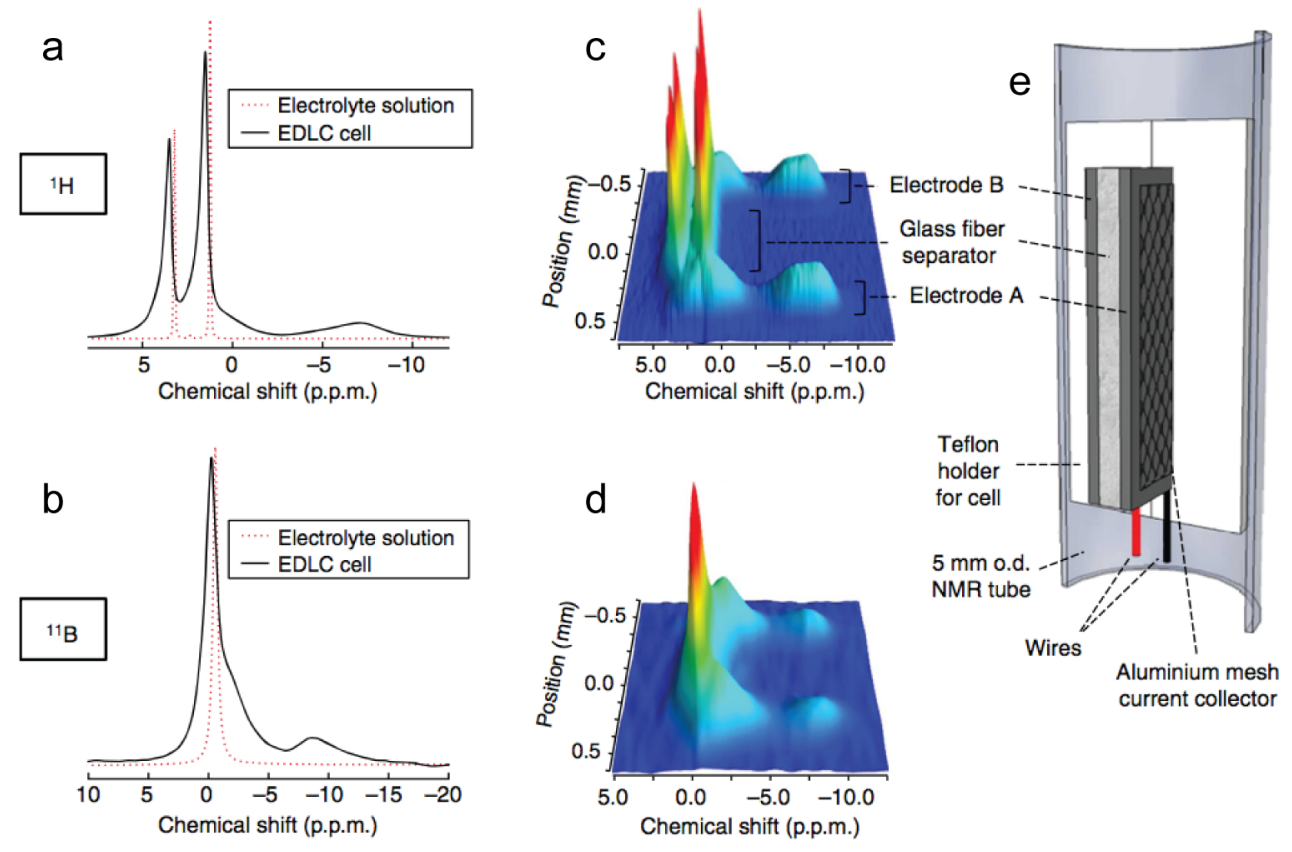

Figure 12. (a) ${ }^{1} \mathrm{H}$ and (b) ${ }^{11} \mathrm{~B}$ NMR spectra extracted from slices through the (c) ${ }^{1} \mathrm{H}$ and (d) ${ }^{11} \mathrm{~B}$ CSIs of a supercapacitor cell comprising YP-50F activated carbon electrodes and $\mathrm{NEt}_{4}-\mathrm{BF}_{4} / \mathrm{ACN}$ electrolyte. NMR spectra of the neat electrolyte are superimposed in (a) and (b). The in situ MRI cell design is illustrated in (e). [Figure reproduced with permission from Ref. $^{77}$. Reprinted by permission from Macmillan Publishers Ltd.: Nature Communications, copyright (2014)].

Illot et al. have exploited the spatial resolution provided by magnetic resonance imaging (MRI) experiments to enable independent yet simultaneous observation of both electrodes. ${ }^{77}$ Using in situ MRI, the electrodes can be studied individually in the standard 'parallel plate' geometry, without the need for the shifted cell design. By using a specially-designed PTFE cell and deuterated solvent, the cations and anions in $\mathrm{NEt}_{4}-\mathrm{BF}_{4} / \mathrm{ACN}$ electrolyte can be studied separately by ${ }^{1} \mathrm{H}$ and ${ }^{11} \mathrm{~B}$ in situ NMR. Conventional NMR spectra for each electrode can be obtained by extracting slices through chemical shift images (CSIs) for the individual electrodes. Although not fully quantitative due to $T_{2}$ dephasing during echo delays used in the experiments, ${ }^{1} \mathrm{H}$ and ${ }^{11} \mathrm{~B}$ CSI slices obtained with the cell held at constant voltages above $0.5 \mathrm{~V}$ show qualitative increases in the cation population in the negative electrode and in the anion population in the positive electrode, indicating that counter-ions are adsorbed onto the carbon surface in both electrodes. Experiments were also performed while a pristine cell was subjected to cyclic voltammetry in order to investigate the charge/discharge dynamics. In the negative electrode, the $\mathrm{CH}_{3}$ chemical shift of the $\mathrm{NEt}_{4}$ cation changed reversibly during charge and discharge. However, in the positive electrode a significant hysteresis was observed, although the chemical shift returned to its original value at $0 \mathrm{~V}$. This should be compared with the ${ }^{19} \mathrm{~F}$ in situ NMR study of the same system by Wang et al., where the ${ }^{19} \mathrm{~F}$ chemical shift of in-pore $\mathrm{BF}_{4}$ anions in the positive and negative electrodes differed by approximately $1 \mathrm{ppm}$ at $0 \mathrm{~V}$. In this case the supercapacitor had been 'pre-cycled' by cyclic 
voltammetry five times prior to the NMR measurement. Illot et al. postulated that the hysteresis observed in the in situ MRI experiments suggests that ion rearrangement during discharge takes place differently in the two electrodes. This could result in irreversible ordering of the ions inside the micropores over multiple cycles, leading to a small amount of charge build up on the electrode surface, which in turn could contribute to ageing of the cell.

\section{Summary and Outlook}

In summary, solid-state NMR has emerged as a powerful tool in the characterization of supercapacitor electrodes and for understanding the charging mechanism. The success of the NMR approach stems from the unique ability to separately observe in-pore ions and solvent molecules from those in bulk electrolyte. These species are subject to ring current effects from carbon surfaces that indiscriminately shield the resonances arising from electrolyte cations, anions and solvent molecules, which allows for their separate observation and quantification. The development of in situ NMR methods whereby working supercapacitors are studied during charging and discharging allow the in-pore populations to be quantified at different charging states, and these measurements have revealed new insights into the molecular mechanisms of charge storage. In particular, solid-state NMR experiments have provided rare experimental proof of the following key points:

1. In general, the pores of the microporous carbon electrodes contain a significant number of electrolyte ions prior to charging.

2. The charging mechanism is not purely absorptive, as has been traditionally assumed, but in most cases involves ion exchange with simultaneous counter-ion adsorption and co-ion desorption from the electrode surface.

Until now, these insights have not been readily available by other experimental techniques, and have important implications for understanding the supercapacitance mechanism. The fact that coions remain inside the micropores during charging means that some of the ionic charge is effectively screened and does not contribute to the capacitance. If future electrode materials could be designed to either favour adsorption of counter-ions or facilitate the ejection of co-ions from the pores during charging, improvements in the energy capacity could be made.

While the NMR approaches that have been developed have already provided lots of new information about supercapacitors, there are many directions for the their future application in the 


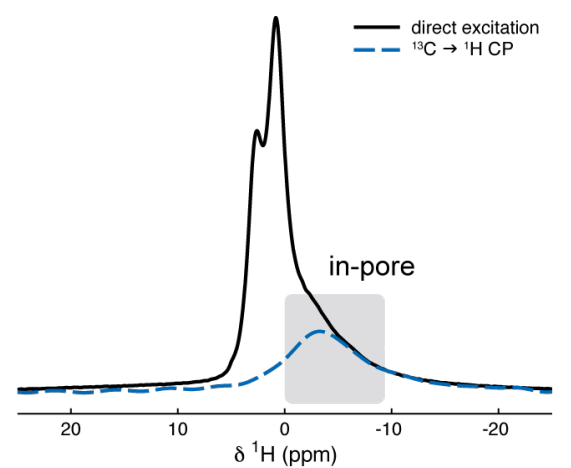

Figure 13. ${ }^{1} \mathrm{H}$ static NMR spectra of a ${ }^{13} \mathrm{C}$-enriched TiC-CDC-1000 electrode film loaded with $\mathrm{NEt}_{4}-\mathrm{BF}_{4} / \mathrm{ACN}$ electrolyte. The spectrum shown by the black line was recorded using a standard single pulse excitation experiment, and the spectrum shown by the blue line was recorded using ${ }^{13} \mathrm{C} \rightarrow{ }^{1} \mathrm{H}$ cross polarization (CP). Only the in-pore resonance is observed in the ${ }^{13} \mathrm{C} \rightarrow{ }^{1} \mathrm{H}$ CP NMR spectrum. [Reprinted from Ref. ${ }^{56}$ with permission from the PCCP Owner Societies].

study of charging mechanisms. Further studies on a wider range of electrolytes are required to gain a more precise understanding of how factors such as relative anion/cation sizes, charge densities and pore size effects influence the charging mechanism. Furthermore, NMR studies that specifically target the solvent molecules are needed to understand the role that the solvent plays in the charge storage process, and to more accurately determine the extent of desolvation inside micropores. While NMR experiments have started to reveal information about ion dynamics in supercapacitor electrodes, more work must be done to understand how the nature of the electrolyte and the structure of the carbon electrodes impact in-pore ionic transport and thus charging/discharging times. Diffusion and relaxation measurements may offer interesting insights here.

There are also opportunities for the development and application of new NMR techniques for the study of supercapacitors. One promising experimental technique is the use of cross polarization (CP) to correlate in-pore species with the carbon surface. Preliminary experiments performed by Forse et al., have already shown it is possible to transfer magnetization from a ${ }^{13} \mathrm{C}$-enriched $\mathrm{TiC}$ $\mathrm{CDC}$ carbon surface to protons in $\mathrm{NEt}_{4}$ cations within the micropores (Figure 12). This approach can be used as a spectral editing tool to 'filter out' the often intense free electrolyte signal, thereby enabling more precise measurements on the in-pore species. The distance dependence of the CP transfer also means that the analysis of $\mathrm{CP}$ build-up curves and $T_{1} \rho$ relaxation times stands to offer important insight into the proximities of electrolyte species to the carbon surface and the dynamics that they undergo inside the micropores.

Further work is also required to better understand the structures of microporous carbons, such that the structures can be correlated with performance in supercapacitors. These materials are challenging to study directly by solid-state NMR, partly owing to the low sensitivity of ${ }^{13} \mathrm{C}$ and other probable defect species such as ${ }^{17} \mathrm{O}$ or ${ }^{14 / 15} \mathrm{~N}$. However, the ongoing development and increasing 
availability of dynamic nuclear polarization (DNP) hardware for signal enhancement in NMR may offer the possibility to study these nuclei directly. Indeed, DNP has already been demonstrated to be a powerful probe of other porous materials due to the fact that the polarization transfer process usually favours observation of solid surfaces rather than the bulk interior. The ability to observe and quantify surface species in microporous carbons using DNP would provide new structural insights into these challenging and complex materials.

\section{Acknowledgements}

The authors acknowledge the Sims Scholarship (A.C.F.), EPSRC (via the Supergen consortium, J.M.G.), and the EU ERC (via an Advanced Fellowship to C.P.G.) for funding. A.C.F. and J.M.G. thank the NanoDTC Cambridge for travel funding. We thank Patrice Simon, Yury Gogotsi, Alexei Kornyshev, Svyatoslav Kondrat and Volker Presser for stimulating discussions and insights into supercapacitors over the course of our research on this subject.

\section{References}

1. Simon, P.; Gogotsi, Y., Materials for electrochemical capacitors. Nature Materials 2008, 7 (11), 845-854.

2. Miller, J. R.; Simon, P., Materials science - Electrochemical capacitors for energy management. Science 2008, 321 (5889), 651-652.

3. Burke, A., R\&D considerations for the performance and application of electrochemical capacitors. Electrochimica Acta 2007, 53 (3), 1083-1091.

4. Augustyn, V.; Simon, P.; Dunn, B., Pseudocapacitive oxide materials for high-rate electrochemical energy storage. Energy \& Environmental Science 2014, 7 (5), 1597-1614.

5. Hu, Y.-Y.; Liu, Z.; Nam, K.-W.; Borkiewicz, O. J.; Cheng, J.; Hua, X.; Dunstan, M. T.; Yu, X.; Wiaderek, K. M.; Du, L.-S.; Chapman, K. W.; Chupas, P. J.; Yang, X.-Q.; Grey, C. P., Origin of additional capacities in metal oxide lithium-ion battery electrodes. Nature Materials 2013, 12 (12), $1130-1136$.

6. Conway, B. E., Electrochemical Capacitors: Scientific, Fundamentals and Technological Applications. Plenum Press: New York, 1999.

7. Kotz, R.; Carlen, M., Principles and applications of electrochemical capacitors. Electrochimica Acta 2000, 45 (15-16), 2483-2498.

8. $\quad$ Simon, P.; Gogotsi, Y., Capacitive Energy Storage in Nanostructured Carbon-Electrolyte Systems. Accounts of Chemical Research 2013, 46 (5), 1094-1103.

[This review provides an explanation of the basic principles of supercapacitors and supercapacitor materials for the non-specialist, and discusses a range of experimental and theoretical insights into the fundamental mechanism]

9. Fic, K.; Lota, G.; Meller, M.; Frackowiak, E., Novel insight into neutral medium as electrolyte for high-voltage supercapacitors. Energy \& Environmental Science 2012, 5 (2), 58425850.

10. Lazzari, M.; Mastragostino, M.; Soavi, F., Capacitance response of carbons in solvent-free ionic liquid electrolytes. Electrochemistry Communications 2007, 9 (7), 1567-1572.

11. Arbizzani, C.; Biso, M.; Cericola, D.; Lazzari, M.; Soavi, F.; Mastragostino, M., Safe, highenergy supercapacitors based on solvent-free ionic liquid electrolytes. Journal of Power Sources 2008, 185 (2), 1575-1579. 
12. Van Aken, K. L.; Beidaghi, M.; Gogotsi, Y., Formulation of Ionic-Liquid Electrolyte To Expand the Voltage Window of Supercapacitors. Angewandte Chemie-International Edition 2015, 54 (16), 4806-4809.

13. Galinski, M.; Lewandowski, A.; Stepniak, I., Ionic liquids as electrolytes. Electrochimica Acta 2006, 51 (26), 5567-5580.

14. Brandt, A.; Pohlmann, S.; Varzi, A.; Balducci, A.; Passerini, S., Ionic liquids in supercapacitors. Mrs Bulletin 2013, 38 (7), 554-559.

15. Van Aken, K. L.; Perez, C. R.; Oh, Y.; Beidaghi, M.; Jeong, Y. J.; Islam, M. F.; Gogotsi, Y., High rate capacitive performance of single-walled carbon nanotube aerogels. Nano Energy 2015, 15, 662-669.

16. Van Aken, K. L.; McDonough, J. K.; Li, S.; Feng, G.; Chathoth, S. M.; Mamontov, E.; Fulvio, P. F.; Cummings, P. T.; Dai, S.; Gogotsi, Y., Effect of cation on diffusion coefficient of ionic liquids at onion-like carbon electrodes. Journal of Physics-Condensed Matter 2014, 26 (28), 10.

17. Zhai, Y.; Dou, Y.; Zhao, D.; Fulvio, P. F.; Mayes, R. T.; Dai, S., Carbon Materials for Chemical Capacitive Energy Storage. Advanced Materials 2011, 23 (42), 4828-4850.

18.* Harris, P. J. F., New perspectives on the structure of graphitic carbons. Critical Reviews in Solid State and Materials Sciences 2005, 30 (4), 235-253.

[This excellent review provides a detailed explanation of the structures of different carbon materials including activated carbons]

19. Kane, M. S.; Goellner, J. F.; Foley, H. C.; DiFrancesco, R.; Billinge, S. J. L.; Allard, L. F., Symmetry breaking in nanostructure development of carbogenic molecular sieves: Effects of morphological pattern formation on oxygen and nitrogen transport. Chemistry of Materials 1996, 8 (8), 2159-2171.

20. Petkov, V.; DiFrancesco, R. G.; Billinge, S. J. L.; Acharya, M.; Foley, H. C., Local structure of nanoporous carbons. Philosophical Magazine B-Physics of Condensed Matter Statistical Mechanics Electronic Optical and Magnetic Properties 1999, 79 (10), 1519-1530.

21. Zetterstrom, P.; Urbonaite, S.; Lindberg, F.; Delaplane, R. G.; Leis, J.; Svensson, G., Reverse Monte Carlo studies of nanoporous carbon from TiC. Journal of Physics-Condensed Matter 2005, 17 (23), 3509-3524.

22. Burian, A.; Dore, J. C., Does carbon prefer flat or curved surfaces? Acta Physica Polonica A 2000, 98 (5), 457-468.

23. Burian, A.; Ratuszna, A.; Dore, J. C.; Howells, S. W., Radial distribution function analysis of the structure of activated carbons. Carbon 1998, 36 (11), 1613-1621.

24. Dash, R.; Chmiola, J.; Yushin, G.; Gogotsi, Y.; Laudisio, G.; Singer, J.; Fischer, J.; Kucheyev, S., Titanium carbide derived nanoporous carbon for energy-related applications. Carbon 2006, 44 (12), 2489-2497.

25. Deschamps, M.; Cadars, S.; Gilbert, E.; Azais, P.; Raymundo-Pinero, E.; Beguin, F.; Massiot, D., A solid-state NMR study of C-70: A model molecule for amorphous carbons. Solid State Nuclear Magnetic Resonance 2012, 42, 81-86.

26. Forse, A. C.; Griffin, J. M.; Presser, V.; Gogotsi, Y.; Grey, C. P., Ring Current Effects: Factors Affecting the NMR Chemical Shift of Molecules Adsorbed on Porous Carbons. Journal of Physical Chemistry C 2014, 118 (14), 7508-7514.

27. Vora, P. M.; Gopu, P.; Rosario-Canales, M.; Perez, C. R.; Gogotsi, Y.; Santiago-Aviles, J. J.; Kikkawa, J. M., Correlating magnetotransport and diamagnetism of sp(2)-bonded carbon networks through the metal-insulator transition. Physical Review B 2011, 84 (15).

28. Palmer, J. C.; Llobet, A.; Yeon, S. H.; Fischer, J. E.; Shi, Y.; Gogotsi, Y.; Gubbins, K. E., Modeling the structural evolution of carbide-derived carbons using quenched molecular dynamics. Carbon 2010, 48 (4), 1116-1123.

29. Takashiro, J.; Kudo, Y.; Kaneko, S.; Takai, K.; Ishii, T.; Kyotani, T.; Enoki, T.; Kiguchi, M., Heat treatment effect on the electronic and magnetic structures of nanographene sheets investigated through electron spectroscopy and conductance measurements. Physical Chemistry Chemical Physics 2014, 16 (16), 7280-7289.

30.* Chmiola, J.; Yushin, G.; Gogotsi, Y.; Portet, C.; Simon, P.; Taberna, P. L., Anomalous increase in carbon capacitance at pore sizes less than 1 nanometer. Science 2006, 313 (5794), 1760-1763.

[This seminal paper was the first work where a pore-size effect on capacitance was identified. This work kick-started much of the recent supercapacitor research that continues today] 
31. Chmiola, J.; Largeot, C.; Taberna, P.-L.; Simon, P.; Gogotsi, Y., Desolvation of ions in subnanometer pores and its effect on capacitance and double-layer theory. Angewandte ChemieInternational Edition 2008, 47 (18), 3392-3395.

32. Lin, R.; Taberna, P. L.; Chmiola, J.; Guay, D.; Gogotsi, Y.; Simon, P., Microelectrode Study of Pore Size, Ion Size, and Solvent Effects on the Charge/Discharge Behavior of Microporous Carbons for Electrical Double-Layer Capacitors. Journal of the Electrochemical Society 2009, 156 (1), A7-A12.

33. Feng, G.; Cummings, P. T., Supercapacitor Capacitance Exhibits Oscillatory Behavior as a Function of Nanopore Size. Journal of Physical Chemistry Letters 2011, 2 (22), 2859-2864.

34. Wu, P.; Huang, J. S.; Meunier, V.; Sumpter, B. G.; Qiao, R., Complex Capacitance Scaling in Ionic Liquids-Filled Nanopores. Acs Nano 2011, 5 (11), 9044-9051.

35. Kondrat, S.; Kornyshev, A., Superionic state in double-layer capacitors with nanoporous electrodes. Journal of Physics-Condensed Matter 2011, 23 (2), 5.

36.* Merlet, C.; Rotenberg, B.; Madden, P. A.; Taberna, P. L.; Simon, P.; Gogotsi, Y.; Salanne, M., On the molecular origin of supercapacitance in nanoporous carbon electrodes. Nature Materials 2012, 11 (4), 306-310.

[This paper describes one of the first theoretical studies that shows the important of ion confinement and rearragement (rather than adsorption/desorption) in the phenomenon of supercapacitance]

37. Merlet, C.; Pean, C.; Rotenberg, B.; Madden, P. A.; Daffos, B.; Taberna, P. L.; Simon, P.; Salanne, M., Highly confined ions store charge more efficiently in supercapacitors. Nature Communications 2013, 4, 6.

38. Kondrat, S.; Wu, P.; Qiao, R.; Kornyshev, A. A., Accelerating charging dynamics in subnanometre pores. Nature Materials 2014, 13 (4), 387-393.

39. Kim, T.; Ideta, K.; Jung, D.; Saito, K.; Park, J.-I.; Rhee, C. K.; Miyawaki, J.; Mochida, I.; Yoon, S.-H., Quantitative analysis of BF4- ions infiltrated into micropores of activated carbon fibers using nuclear magnetic resonance. RSC Advances 2014, 4 (32), 16726-16730.

40. Kolodziejski, W.; Klinowski, J., Kinetics of Cross-Polarization in Solid-State NMR: A Guide for Chemists. Chemical Reviews 2002, 102 (3), 613-628.

41.* Deschamps, M.; Gilbert, E.; Azais, P.; Raymundo-Pinero, E.; Ammar, M. R.; Simon, P.; Massiot, D.; Beguin, F., Exploring electrolyte organization in supercapacitor electrodes with solidstate NMR. Nature Materials 2013, 12 (4), 351-358.

[This is the first example of the application of ex situ NMR for the study of supercapacitors]

42. Vold, R. L.; Hoatson, G. L., Effects of jump dynamics on solid state nuclear magnetic resonance line shapes and spin relaxation times. Journal of Magnetic Resonance 2009, 198 (1), 57-72.

43. Griffin, J. M.; Forse, A. C.; Wang, H.; Trease, N. M.; Taberna, P. L.; Simon, P.; Grey, C. P., Ion counting in supercapacitor electrodes using NMR spectroscopy. Faraday Discussions 2014, 176, 49-68.

44. Dickinson, L. M.; Harris, R. K.; Shaw, J. A.; Chinn, M.; Norman, P. R., Oxygen-17 and deuterium NMR investigation into the adsorption of water on activated carbon. Magnetic Resonance in Chemistry 2000, 38 (11), 918-924.

45. Harris, R. K.; Thompson, T. V.; Forshaw, P.; Foley, N.; Thomas, K. M.; Norman, P. R.; Pottage, C., A magic-angle spinning NMR study into the adsorption of deuterated water by activated carbon. Carbon 1996, 34 (10), 1275-1279.

46. Harris, R. K.; Thompson, T. V.; Norman, P. R.; Pottage, C., Adsorption competition onto activated carbon, studied by magic-angle spinning NMR. Journal of the Chemical Society-Faraday Transactions 1996, 92 (14), 2615-2618.

47. Harris, R. K.; Thompson, T. V.; Norman, P. R.; Pottage, C., Phosphorus-31 NMR studies of adsorption onto activated carbon. Carbon 1999, 37 (9), 1425-1430.

48. Shaw, J. A.; Harris, R. K.; Norman, P. R., Magic-angle spinning NMR study of the competitive adsorption of an organophosphate and an organophosphonate on activated carbon. Langmuir 1998, 14 (23), 6716-6721.

49. Sekhaneh, W.; Kotecha, M.; Dettlaff-Weglikowska, U.; Veeman, W. S., High resolution NMR of water absorbed in single-wall carbon nanotubes. Chemical Physics Letters 2006, 428 (13), 143-147. 
50. Liu, X.; Pan, X.; Shen, W.; Ren, P.; Han, X.; Bao, X., NMR Study of Preferential Endohedral Adsorption of Methanol in Multiwalled Carbon Nanotubes. Journal of Physical Chemistry C 2012, 116 (14), 7803-7809.

51. Lazzeretti, P., Ring currents. Progress in Nuclear Magnetic Resonance Spectroscopy 2000, $36(1), 1-88$.

52.* Anderson, R. J.; McNicholas, T. P.; Kleinhammes, A.; Wang, A.; Liu, J.; Wu, Y., NMR Methods for Characterizing the Pore Structures and Hydrogen Storage Properties of Microporous Carbons. Journal of the American Chemical Society 2010, 132 (25), 8618-8626.

[This excellent paper discusses the phenomenological effects of ring currents on the NMR frequencies of species adsorbed on porous carbon, and how they can be used to derive information about the carbon structure]

53. N. Ganguli, K. S. K., The Magnetic and other properties of the free electrons in graphite. Proceedings of the Royal Society of London, Series A 1941, 177, 168-182.

54.* Presser, V.; Heon, M.; Gogotsi, Y., Carbide-Derived Carbons - From Porous Networks to Nanotubes and Graphene. Advanced Functional Materials 2011, 21 (5), 810-833.

[This paper gives a detailed overview of the synthesis and properties of metal carbide-derived carbons which are widely used in studies of supercapacitor charging mechanisms]

55. Laudisio, G.; Dash, R. K.; Singer, J. P.; Yushin, G.; Gogotsi, Y.; Fischer, J. E., Carbidederived carbons: A comparative study of porosity based on small-angle scattering and adsorption isotherms. Langmuir 2006, 22 (21), 8945-8950.

56. Forse, A. C.; Griffin, J. M.; Wang, H.; Trease, N. M.; Presser, V.; Gogotsi, Y.; Simon, P.; Grey, C. P., Nuclear magnetic resonance study of ion adsorption on microporous carbide-derived carbon. Physical Chemistry Chemical Physics 2013, 15 (20), 7722-7730.

57. Borchardt, L.; Oschatz, M.; Paasch, S.; Kaskel, S.; Brunner, E., Interaction of electrolyte molecules with carbon materials of well-defined porosity: characterization by solid-state NMR spectroscopy. Physical Chemistry Chemical Physics 2013, 15 (36), 15177-15184.

58. Forse, A. C.; Merlet, C.; Allan, P. K.; Humphreys, E. K.; Griffin, J. M.; Aslan, M.; Zeiger, M.; Presser, V.; Gogotsi, Y.; Grey, C. P., New Insights into the Structure of Nanoporous Carbons from NMR, Raman, and Pair Distribution Function Analysis. Chemistry of Materials 2015, 27 (19), 68486857.

59. Chen, Z. F.; Wannere, C. S.; Corminboeuf, C.; Puchta, R.; Schleyer, P. V., Nucleusindependent chemical shifts (NICS) as an aromaticity criterion. Chemical Reviews 2005, 105 (10), 3842-3888.

60. Kibalchenko, M.; Payne, M. C.; Yates, J. R., Magnetic Response of Single-Walled Carbon Nanotubes Induced by an External Magnetic Field. Acs Nano 2011, 5 (1), 537-545.

61. Ren, P.; Zheng, A.; Pan, X.; Han, X.; Bao, X., DFT Study on the NMR Chemical Shifts of Molecules Confined in Carbon Nanotubes. Journal of Physical Chemistry C 2013, 117 (44), 2341823424.

62. Ren, P.; Zheng, A.; Xiao, J.; Pan, X.; Bao, X., Exploring the ring current of carbon nanotubes by first-principles calculations. Chemical Science 2015, 6 (2), 902-908.

63. Buhl, M., The relation between endohedral chemical shifts and local aromaticities in fullerenes. Chemistry-a European Journal 1998, 4 (4), 734-739.

64. Sumanasekera, G. U.; Chen, G.; Takai, K.; Joly, J.; Kobayashi, N.; Enoki, T.; Eklund, P. C., Charge transfer and weak chemisorption of oxygen molecules in nanoporous carbon consisting of a disordered network of nanographene sheets. Journal of Physics-Condensed Matter 2010, 22 (33), 8.

65. Merlet, C.; Forse, A. C.; Griffin, J. M.; Frenkel, D.; Grey, C. P., Lattice simulation method to model diffusion and NMR spectra in porous materials. Journal of Chemical Physics 2015, 142 (9).

66.* Wang, H.; Forse, A. C.; Griffin, J. M.; Trease, N. M.; Trognko, L.; Taberna, P.-L.; Simon, P.; Grey, C. P., In Situ NMR Spectroscopy of Supercapacitors: Insight into the Charge Storage Mechanism. Journal of the American Chemical Society 2013, 135 (50), 18968-18980.

[This paper gives a detailed overview practical considerations and experimental observables in in situ NMR spectroscopy of supercapacitors]

67. Oth, J. F. M.; Woo, E. P.; Sondheim.F, DIANION OF 18 ANNULENE. Journal of the American Chemical Society 1973, 95 (22), 7337-7345. 
68. Forse, A. C.; Griffin, J. M.; Merlet, C.; Bayley, P. M.; Wang, H.; Simon, P.; Grey, C. P., NMR Study of Ion Dynamics and Charge Storage in Ionic Liquid Supercapacitors. Journal of the American Chemical Society 2015, 137 (22), 7231-7242.

69. Fukano, M.; Fujimori, T.; Segalini, J.; Iwama, E.; Taberna, P.-L.; liyama, T.; Ohba, T.; Kanoh, H.; Gogotsi, Y.; Simon, P.; Kaneko, K., Vertically Oriented Propylene Carbonate Molecules and Tetraethyl Ammonium Ions in Carbon Slit Pores. Journal of Physical Chemistry C 2013, 117 (11), 5752-5757.

70.* Trease, N. M.; Zhou, L.; Chang, H. J.; Zhu, B. Y.; Grey, C. P., In situ NMR of lithium ion batteries: Bulk susceptibility effects and practical considerations. Solid State Nuclear Magnetic Resonance 2012, 42, 62-70.

[Although this paper does not concern supercapacitors, it gives a detailed overview of the practical aspects of in situ NMR spectroscopy]

71. Wang, H.; Koster, T. K. J.; Trease, N. M.; Segalini, J.; Taberna, P. L.; Simon, P.; Gogotsi, Y.; Grey, C. P., Real-Time NMR Studies of Electrochemical Double-Layer Capacitors. Journal of the American Chemical Society 2011, 133 (48), 19270-19273.

72. D. G. Cory, W. M. R., Suppression of Signals from the Probe in Bloch Decay Spectra. Journal of Magnetic Resonance 1988, 80, 128-132.

73. Levi, M. D.; Levy, N.; Sigalov, S.; Salitra, G.; Aurbach, D.; Maier, J., Electrochemical Quartz Crystal Microbalance (EQCM) Studies of lons and Solvents Insertion into Highly Porous Activated Carbons. Journal of the American Chemical Society 2010, 132 (38), 13220-13222.

74.* $\quad$ Griffin, J. M.; Forse, A. C.; Tsai, W.-Y.; Taberna, P.-L.; Simon, P.; Grey, C. P., In situ NMR and electrochemical quartz crystal microbalance techniques reveal the structure of the electrical double layer in supercapacitors. Nature Materials 2015, 14 (8), 812.

[In this paper in situ NMR is used for the first time to simultaneously quantify anions and cations, thereby giving a full picture of the charging mechanism]

75. Luo, Z.-X.; Xing, Y.-Z.; Ling, Y.-C.; Kleinhammes, A.; Wu, Y., Electroneutrality breakdown and specific ion effects in nanoconfined aqueous electrolytes observed by NMR. Nature Communications 2015, 6.

76. Luo, Z.-X.; Xing, Y.-Z.; Liu, S.; Ling, Y.-C.; Kleinhammes, A.; Wu, Y., Dehydration of lons in Voltage-Gated Carbon Nanopores Observed by in Situ NMR. The Journal of Physical Chemistry Letters 2015, 6 (24), 5022-5026.

77.* Ilott, A. J.; Trease, N. M.; Grey, C. P.; Jerschow, A., Multinuclear in situ magnetic resonance imaging of electrochemical double-layer capacitors. Nature Communications 2014, 5.

[This is the first application of magnetic resonance imaging to supercapacitors] 


\section{Author Information}

John Griffin studied for his undergraduate degree in physics at the University of Warwick, U.K., and remained there to carry out a Ph.D. under the supervision of Professor Steven Brown. In 2008, John joined the group of Dr. Sharon Ashbrook at the University of St. Andrews to pursue postdoctoral research in the field of experimental and computational methods in NMR spectroscopy. In 2012 he moved to the University of Cambridge to work in the research group of Prof. Clare Grey with a particular focus on supercapacitors. In 2015 he took up a lectureship in materials chemistry at Lancaster University. His research interests are centered around applications and development of advanced solid-state NMR methods to study inorganic systems and materials.

Alexander Forse studied Natural Sciences (M.Sc.) at the University of Cambridge, U.K. He continued in Cambridge carrying out graduate research in the group of Prof. Clare Grey in the Department of Chemistry, and he received his Ph.D. in 2015 for work on the development and application of NMR spectroscopy to the study of supercapacitors. Alex's general research interests centre around the application of magnetic resonance methods to study materials for energy storage and production, with current postdoctoral research focussing on understanding electrolyte ion transport in porous electrode materials.

Clare Grey is the Geoffrey Moorhouse-Gibson Professor of Chemistry at Cambridge University and a Fellow of Pembroke College. She received a BA and D. Phil. (1991) in Chemistry from the University of Oxford. After post-doctoral fellowships in the Netherlands and at DuPont CR\&D in Wilmington, DE, she joined the faculty at Stony Brook University (SBU) as an Assistant (1994), Associate (1997) and then Full Professor (2001-2015). She moved to Cambridge in 2009, maintaining an adjunct position at SBU. Her recent honors and awards include the 2011 Royal Society Kavli Lecture and Medal for work relating to the Environment/Energy, Honorary PhD Degrees from the Universities of Orleans (2012) and Lancaster (2013), the Gunther Laukien Award from the Experimental NMR Conference (2013), the Research Award from the International Battery Association (2013), the Royal Society Davy Award (2014) and the Arfvedson-Schlenk Prize from the German Chemical Society (2015). She is a Fellow of the Royal Society. Her current research interests include the use of solid state NMR and diffraction-based methods to determine structurefunction relationships in materials for energy storage (batteries and supercapacitors), conversion (fuel cells) and carbon capture. 